EUROPEAN ORGANIZATION FOR NUCLEAR RESEARCH

CERN-PPE/94-114

October 31, 1994

\title{
SEARCHES FOR NEW PARTICLES
}

D. Treille

CERN, Geneva, Switzerland 
The Standard Model (SM) of particle physics summarizes what we consider presently to be the truth about the elementary building blocks of matter and their interactions (see Appendix 1).

In brief, these building blocks are fermions ( spin 1/2), which appear as structureless with the resolution power presently accessible (a per mil of a fermi), grouped into three families, and, within a family, into two very different species: leptons, which propagate freely in space-time, and quarks, which seem inescapably bound within nucleons and manifest themselves only as jets of ordinary hadrons. Left-handed fermions (right-handed antifermions) appear as doublets of a new quantum number, the weak isospin. Quarks carry a further quantum number, the colour, specific to strong interactions, and appear as three species, corresponding to three different values of the colour or, metaphorically, to 'three colours'.

The fermions of the first family are sufficient to build the present universe, including ourselves. A very obvious question concerns the raison d'être of the two other families and the actual number of such replica.

Of the 12 basic fermions, two - the heaviest quark called the top $(\mathrm{t})$ and the neutrino of the 3rd family $\left(\nu_{\tau}\right)$-have not been observed directly ${ }^{*}$; filling this lacuna is the first priority of particle physics, although one has no real doubt about their existence.

This set of elementary building blocks certainly represents a remarkable reduction à la Mendeleiev compared to the crowded world of hundreds of hadrons which prevailed three decades ago. It is nevertheless still complicated.

On the other hand the way fermions interact, through the exchange of bosons, is rather simple and universal, in all types of interactions. The questions concern the level of unification of these interactions: strictly speaking, the SM only claims to describe the unification of electromagnetic and weak ones.

Within its frame of applicability the SM has up to now accounted qualitatively and quantitatively for all phenomena and measurements.

It is, however, commonplace to say that, in spite of its successes, the SM is largely unsatisfactory. It should first be remembered that up to now it has only been partially tested: whilst the couplings of bosons to fermions have been well studied, the couplings of electroweak bosons to themselves have not yet been checked. The scalar sector, describing the potential existence and role of scalar particles, in particular the SM Higgs boson, is still a pure assumption.

Even if the SM successfully passes these further tests, it will be left with its defects and insufficiencies. The SM has 18 free parameters, to which at least one, $\theta_{Q C D}$ (see Appendix 1), has to be added, and it would have even more if the assumptions of three families only and zero neutrino mass are relaxed.

Although it is a renormalizable theory, the SM, in its description of physics, has deficiencies. One is the instability of the mass of an elementary scalar, such as the Higgs boson, under radiative corrections in the presence of a high scale, for instance Planck's scale. Another one is the so-called triviality of the coupling in the scalar sector.

Finally, the SM does not explain and even does not address basic questions such as the replication of families, and the observed mass spectra.

On the other hand, the considerable strength of the SM and the big chance it offers to contemporary high-energy physics is that, within its domain of application, it is a

*) See however the footnote in Section 4.4 
computable theory to a level of accuracy limited by technical rather than by fundamental considerations.

The usual ways to cure, at least partly, the defects of the SM consist in one of the following two methods, which both call for the existence of new particles and/or effects:

i) To require more symmetry, and to impose from the start relationships between different particles or different interactions.

Obvious symmetries that one could try to restore are the symmetry between leftand right-handed objects, and the symmetry between quarks and leptons, etc.

The attempt to relate the three basic coupling constants of the different forces present in the SM leads to the idea of the Grand Unification Theory (GUT) (Georgi 74, Langacker 81, Ross 84).

Supersymmetry (SUSY) (Appendix 2) (Fayet 77, Nilles 84, Haber 85), which establishes a relationship between fermions and bosons, provides a way to solve the mass stability problem by cancelling divergences between the two spin species: it is a broken symmetry since none of the new states it calls for have yet been observed. The search for these new particles, which, with good arguments, are considered to be not too heavy, is also a priority of high-energy physics.

The running of the coupling constants of the three interactions, and their apparent convergence at very high energy in the supersymmetric case (Ellis 90, Langacker 91a, Amaldi 91, Anselmo 91), lead us to consider the GUT idea as a likely possibility. Such hints do not constitute a proof, but are interesting enough to place SUSY ahead of other candidates for a theory beyond the SM, and a large place will be given to it in this review. However, even if it is correct, it will still fail to explain some burning questions.

ii) To introduce some underlying structure or dynamics.

This is the best way of explaining facts such as the replication of families and mass spectra. But all such theories still have, to various extents, conceptual problems and/or unwanted consequences (Lyons 83, Harari 84, Peskin 85).

An example of such a theory, limited to the scalar sector, is technicolour (Weinberg 79, Susskind 79, Fahri 81), which postulates, with many variants, the existence of new fermions and interactions: the Higgs bosons are replaced by bound states of these fermions.

A few cases will be discussed, but only from a phenomenological point of view, and without considering the difficulties of the theory itself.

The search for new particles is a very broad subject. I will limit myself essentially to searches at accelerators, already existing or under study.

\section{The Machines, Present and Future}

The ongoing programme of the Fermilab Tevatron, colliding protons and antiprotons, its expected evolution, and its promises for the discovery of top, and for other search channels, are described in Barbaro Galtieri 93.

Until recently two large hadron colliders were considered for the future: the SSC (Superconducting Super Synchrotron) in Texas, and the LHC (Large Hadron Collider) at CERN. The LHC, after the negative decision about the SSC, is the only one left, and represents the only chance to reach, in ten years or so, the multi-TeV region for scattering fundamental constituents in hadronic collisions. The LHC parameters are:

- a centre-of-mass energy of $14(2 \times 7) \mathrm{TeV}$;

- a luminosity reaching $10^{34} \mathrm{~cm}^{-2} \mathrm{~s}^{-1}$. 
The main technical challenges are, for the machine, the realization of a large set of high field superconducting magnets and, for the experiment, the ability to exploit this very high luminosity.

The rich potential of future large hadron colliders (HC) is presented in detail in Virdee 93, Pauss 94, and in the LHC Letters of Intent (Atlas 92, CMS92, L3P92).

The e- $\mathrm{p}$ collider HERA recently produced its first physics results (Wolf 94). With the expected increase of its luminosity, it will allow the exploration of many physics channels, relevant for instance to the composite models.

Concerning $\mathrm{e}^{+} \mathrm{e}^{-}$colliders, low-energy ones continue to provide accurate measurements about heavy fermions: BEPC in Beijing obtained an ultraprecise value of $m_{\tau}$ (Bai 92). CESR in Cornell is obtaining precious information on b-flavoured objects (Besson 94).

Projects towards a genuine threshold beauty factory, asymmetric in energy and aiming to observe $\mathrm{CP}$ violation in the $\mathrm{B}^{0}$ sector on the $\mathrm{Y}(4 \mathrm{~S})$, are on their way (Zisman 94, Iwata 94): the foreseen luminosity is in excess of $10^{33} \mathrm{~cm}^{-2} \mathrm{~s}^{-1}$.

At high energies TRISTAN and especially the Large Electron-Positron storage ring (LEP) and the SLAC Linear Collider (SLC) are producing a harvest of results on electroweak physics and searches for new objects. The SLC has reached a high level of $\mathrm{e}^{-}$ longitudinal polarization ( $60 \%$, with $80 \%$ expected) which will be exploited in the coming years. LEP I with its four experiments has already registered $\sim 8,000,000 \mathrm{Z}^{0}$ decays and will, in the next two years, increase its statistics of the $Z^{0}$. The following step will be to raise its energy, first to $\sim 175 \mathrm{GeV}$ c.m. and hopefully later to $\sim 200 \mathrm{GeV}$. In this note, for illustration, we will mention the physics potential at $190 \mathrm{GeV}$ and $210 \mathrm{GeV}$ (210 GeV being the maximum energy one can hope to reach by adding accelerating radiofrequency (RF) cavities without further civil engineering work). A longitudinal polarization programme (Alexander 88) and a programme of higher luminosities (Blucher 91) have been discussed as possibilities for the future of LEP.

$\mathrm{e}^{+} \mathrm{e}^{-}$linear colliders (LC) will take over from circular ones after the advent of LEP200. A $\sqrt{s} \sim 500 \mathrm{GeV}$ version is being studied (JLC 92, Wiik 93, Guignard 93, Siemann 94): we shall name it here the Next Linear Collider (NLC). As we shall see, many physics arguments call for higher energies, to which the NLC must be expandable. We will assume a luminosity of $10^{33} \mathrm{~s} /(500)^{2} \mathrm{~cm}^{-2} \mathrm{~s}^{-1}$ for these machines, i.e. $10 \mathrm{fb}^{-1}$ per year at the NLC, $\sim 10$ times more at $1.5 \mathrm{TeV}$.

Linear colliders can also be used in the $\mathrm{e}^{-} \mathrm{e}^{-}$mode (Heusch 93) and in the $\gamma \gamma$ mode by backscattering laser beams from the $\mathrm{e}^{ \pm}$beams (Ginzburg 82). For LCs, and a fortiori for the $\gamma \gamma$ mode, a long programme of technical developments is still needed.

In HCs of high luminosity, the main experimental problem is due to the large rate of hadronic interactions, leading to high levels of occupancy and irradiation. A vigorous R\&D programme on the new experimental techniques required for LHCs has been launched: this is excellently described in Iarocci 94. With linear colliders the situation should be less severe, although a strong background of soft $\mathrm{e}^{ \pm}$and some level of underlying $\gamma \gamma$ hadronic events will be present owing to bremsstrahlung and beamstrahlung phenomena (Chen 93).

\section{$3 \quad$ Indirect and Direct Searches}

The successes of the SM are quantitative ones: observables computed from the SM basic input elements agree with the measurements to a high level of accuracy. Conversely, any discrepancy - which did not show up until now except possibly for the result presented 
in 4.3.1-would reveal some inadequacy of the SM. From a set of such discrepancies it would even be possible to attempt to tell what is the origin of the new physics. This is the idea of indirect searches that we develop in Section 4. An advantage is that, in many possible cases of new physics, the discrepancy can be felt well below the threshold for actually producing it.

However, the classical method of search is to produce the new phenomenon and observe it directly. One looks for topologies of final states which cannot be present, or are present below some level, within the frame of the Standard Model. A simple example would be the presence of acollinear, acoplanar lepton pairs in the $Z^{0}$ decay at LEP. For the difficult cases, the nature of the collisions, as well as the quality of the machine and of the detector are crucial, as we shall see.

Other features of the event, such as manifestations of heavy flavours (b-tagging), are also starting to be exploited for searches, and will certainly be a key for future programmes.

\section{$4 \quad$ Indirect Searches}

\subsection{Principle of Indirect Searches}

A measurement has to be compared to an expectation. To compute an observable in the SM one needs the input of the three basic electroweak parameters (Appendix 1)

$$
g \quad g^{\prime} \quad v
$$

which are actually replaced by the equivalent set of three well-determined quantities

$$
\alpha \quad G_{\mu} \quad m_{Z},
$$

the fine structure constant, the muon decay constant, and the $\mathrm{Z}^{0}$ mass, respectively. At tree level, the set $\left(\alpha, G_{\mu}, m_{Z}\right)$ would be enough to compute any leptonic observable. However, the intervention of the SM particles, in particular the top and Higgs, through virtual loop effects, changes the observable value and, in the ignorance of their mass, it is only possible to adopt for it a reference value. For hadronic processes, the strong coupling $\alpha_{s}$ intervenes as well. In summary, for the observable $O$, it is necessary to compute:

$$
O\left(\alpha, G_{\mu}, m_{Z}, m_{\text {top }}^{\text {ref }}, m_{\text {Higgs }}^{\text {ref }}, \alpha_{s}, \ldots\right)
$$

and the goal is to measure an eventual discrepancy:

$$
\Delta O\left(m_{\text {top }}, m_{\text {Higgs }}, \ldots, \text { new physics }\right),
$$

which could be due to a departure of the top and Higgs masses from their reference value, or to new physics. $\Delta O$ should in any case be a small quantity, and this fact calls for the best possible accuracies, both for the experiment and for the theoretical estimate.

Actually the largest virtual effect by far is due to the top: up to now accurate measurements at LEP I have essentially been used as indicators of the top mass. The latest combined results from LEP, the SLC and other fields (neutrino scattering, $m_{W} / m_{Z}$ ) give (LEP 94)

$$
m_{t}=177_{-11}^{+11}{ }_{-19}^{+18} \mathrm{GeV} .
$$

The central value from LEP alone is $m_{t}=172 \mathrm{GeV}$. The second error is due to the Higgs mass variation, and the lowest value is about that expected in the case of a light Higgs, as in the Minimal Standard Supersymmetric Model (MSSM).

Once the direct measurement of $m_{t}$ is performed (see Section 4.4) the sensitivity to smaller effects will be dramatically increased. 


\subsection{Impact of the $\mathrm{Z}$ Line Shape}

A very accurate set of measurements, at LEP and the SLC, have provided the $Z$ line shape in great detail (Lefrançois 93 ). The $Z^{0}$ mass, at present known to $\pm 4 \mathrm{MeV}$, within the SM frame, became the third basic input quantity of the SM. The $Z^{0}$ production crosssection, measured to $\sim 1 / 2 \%$, has shown that only three families of fermions with a light neutrino exist. The $Z^{0}$ total width, $\Gamma_{Z}$, and its invisible width, $\Gamma_{i n v}$, known respectively to $\pm 4 \mathrm{MeV}$ and $\pm 3.5 \mathrm{MeV}$, allowed, by comparison with the SM expectation, limits to be set on the existence of a large number of possible new particles which could appear in $\mathrm{Z}^{0}$ decay. Table 1 , and the comments accompanying it, summarize the situation at LEP (Giacomelli 93). Although this method allows some possibilities to be ruled out up to the kinematic limit, it only has a limited impact in more difficult cases, and direct searches are mandatory to pursue and improve the exploration.

\subsection{The Best Observables}

An illustration of the sensitivity of the electroweak observables to various deviations from the SM is given in Langacker 91 . Figure 1 shows the sensitivity of the various electroweak observables to a departure from an assumed starting point, the SM with $m_{t}$ $=100 \mathrm{GeV}$, and $m_{H}=100 \mathrm{GeV}$.

Figure 1a shows the sensitivity to a shift in $m_{t}(200 \mathrm{GeV}$ instead of 100); Fig. $1 b$ gives the pattern for the adjunction of special new particles expected in composite models. Figure 1a is quite typical of most of the likely departures; Fig. 1b tells us that, in some particular cases, low-energy observables, such as those from atomic parity violation (Bouchiat 84, Gilbert 86), can play a key role as well. Let us focus on two of the most efficient ones, and introduce useful combinations of them.

\subsection{1 $A_{L R}$}

This is the spin asymmetry, obtained simply by comparing the $Z^{0}$ production crosssection from left- and right-handed $\mathrm{e}^{-}$in $\mathrm{e}^{+} \mathrm{e}^{-}$collisions.

Its sensitivity to $\sin ^{2} \theta_{W}$ (the electroweak mixing angle, Appendix 1), its low level of detector systematics, and good statistical conditions (all $Z^{0}$ final states can be used), combined, in LEP with a 'free' and accurate beam polarization measurement, make it the 'queen' of electroweak observables (Prescott 80, Alexander 88).

Its potential, as a function of the number of registered $Z^{0}$ and for various accuracies of the polarization measurement, is shown in Fig. 2. The possible performance of LEP is indicated. However, longitudinal polarization at LEP has a low priority in the ongoing programme.

The results will therefore come from the SLC. The arrow in Fig. 2 shows their published result (Abe 93a) obtained with a polarization $P_{e}$ of $\sim 24 \%$. It should be superseded soon by much better values since the polarization has been raised to $60 \%$, and will later reach $\sim 80 \%$. A recent value (Abe 94) from 1993 data leads to:

$$
\sin ^{2} \theta_{W}=0.2292 \pm 0.0010
$$

This is $2.6 \sigma$ below the LEP average, $\sin ^{2} \theta_{W}^{L E P}=0.2322 \pm 0.0005$ (LEP 94). Interpreted within the SM, the SLC value would correspond to $m_{t} \cong 250 \mathrm{GeV}$. This discrepancy, not yet worrysome as such, may, if it is confirmed, be the first problem encountered in testing the SM. At SLAC the foreseable accuracy will depend on the knowledge of the polarization, and could correspond, for $\Delta P / P=1 \%$, to: $\Delta \sin ^{2} \theta_{W} \simeq 0.0003$, similar to the potential reach of LEP. 
Table 1

Particle mass limits from LEP I [from (Giacomelli 93)]

\begin{tabular}{|c|c|c|c|}
\hline Particle & Symbol & $\begin{array}{l}\text { Mass limit } \\
\quad(\mathrm{GeV})\end{array}$ & Comments \\
\hline $\begin{array}{l}\text { Top quark } \\
\tau \text { neutrino } \\
\text { 4th generation charged lepton } \\
\text { 4th generation neutral lepton } \\
\text { 4th generation down-type quark } \\
\text { Isosinglet neutral heavy lepton }\end{array}$ & $\begin{array}{c}\mathrm{t}_{\tau} \\
\mathrm{L}^{ \pm} \\
\mathrm{L}^{0} \\
\mathrm{~b}^{\prime} \\
\mathrm{N}_{\ell}\end{array}$ & $\begin{array}{l}m_{Z^{0}} / 2 \\
\quad- \\
m_{Z^{0}} / 2 \\
m_{Z^{0}} / 2 \\
m_{Z^{0}} / 2 \\
m_{Z^{0}}\end{array}$ & $\begin{array}{l}\text { From } Z^{0} \text { data }^{*)}: m_{t} \sim 170 \mathrm{GeV} \\
\text { No limits from LEP } \\
\text { Only } 3 \text { families from } Z^{0} \text { width }\end{array}$ \\
\hline $\begin{array}{l}\text { Charged sleptons } \\
\text { Sneutrinos } \\
\text { Charginos } \\
\text { Scalar quarks } \\
\text { Neutralinos } \\
\text { Gluinos }\end{array}$ & $\begin{array}{c}\tilde{\ell}^{ \pm} \\
\widetilde{\nu} \\
\tilde{\chi}^{ \pm} \\
\widetilde{q} \\
\tilde{\chi}^{0} / \widetilde{\chi}^{0^{\prime}} \\
\widetilde{g}\end{array}$ & $\begin{array}{c}m_{Z^{0}} / 2 \\
37 \\
m_{Z^{0}} / 2 \\
m_{Z^{0}} / 2 \\
18.4 / 45 \\
-\end{array}$ & $\begin{array}{l}\text { Pair production } \\
41.7 \mathrm{GeV} \text { if triple mass degeneracy } \\
\text { Pair production } \\
\text { Theory }+ \text { MSSM }+ \text { GUT }+\ldots\end{array}$ \\
\hline Photinos & $\tilde{\gamma}$ & & R-parity violation: see Section 12 \\
\hline $\begin{array}{l}\text { Standard Model Higgs } \\
\text { SUSY Higgses } \\
\text { Doubly charged Higgs }\end{array}$ & $\begin{array}{c}H_{S M}^{0} \\
h^{0} \\
A^{0} \\
H^{ \pm} \\
H^{ \pm \pm}\end{array}$ & $\begin{array}{c}63 \\
42 \\
\sim 30 \\
m_{Z^{0}} / 2 \\
m_{Z^{0}} / 2\end{array}$ & $\begin{array}{l}\text { MSSM-DELPHI limit } \\
\text { MSSM-DELPHI limit } \\
\text { Pair production } \\
\text { Pair production }\end{array}$ \\
\hline $\begin{array}{l}\text { Excited charged leptons }\left(\mathrm{e}^{*}, \mu^{*}, \tau^{*}\right) \\
\text { Excited charged leptons } \\
\text { Excited neutrinos } \\
\text { Excited neutrinos } \\
\text { Excited quarks }\end{array}$ & $\begin{array}{l}\ell^{*} \\
\nu^{*} \\
q^{*}\end{array}$ & $\begin{array}{c}m_{Z^{0}} / 2 \\
f / \lambda<10^{-3} \\
m_{Z^{0}} / 2 \\
m_{Z^{0}} \\
m_{Z^{0}}\end{array}$ & $\begin{array}{l}\text { Pair production } \\
\text { For } m_{Z^{0}} / 2<m_{\ell^{*}}<m_{Z^{0}} \\
\text { Pair production } \\
\text { Single production } \\
\text { Single production }\end{array}$ \\
\hline Leptoquarks & $\mathrm{D}$ & $m_{Z^{0}} / 2(77)$ & Pair (single) production \\
\hline New heavy gauge bosons & $Z^{\prime}$ & 100 & From $Z^{0}$ line shape and models \\
\hline $\begin{array}{l}\text { Free quarks } \\
\text { Magnetic monopoles }\end{array}$ & $\begin{array}{c}q \\
M M\end{array}$ & $\begin{array}{c}43 \\
m_{Z^{\circ}} / 2\end{array}$ & $\begin{array}{l}\text { From } \mathrm{d} E / \mathrm{d} x \text { in TPC of ALEPH } \\
\text { Nuclear track detectors }\end{array}$ \\
\hline
\end{tabular}

*) and preliminary direct evidence from CDF 
LEP I, from the measurement of $Z^{0}$ observables, and the SLC, from $A_{L R}$, should bring the uncertainty on $\sin ^{2} \theta_{W}$ down to \pm 0.0003 .

\subsection{2 $m_{W}$}

From the HC experiments (CDF and DO at Fermilab, UA2 at CERN) the present result of the direct measurement is:

$$
m_{W}=80.23 \pm 0.18 \mathrm{GeV}
$$

Within the SM frame, one gets from LEP accurate measurements the indirect result:

$$
m_{W}=80.28 \pm 0.08 \mathrm{GeV} .
$$

Both values agree very well. As in the case of the top mass, this seems to favour the LEP result on $\sin ^{2} \theta_{W}$.

The Fermilab Collider will probably reach $\Delta m_{W}= \pm 100 \mathrm{MeV}$.

In a LEPC workshop (Camilleri 92) it was shown that by using the reconstruction/rescaling method in the channel $\mathrm{e}^{+} \mathrm{e}^{-} \rightarrow W^{+} W^{-}$, each of the LEP experiments at LEP200 should reach, for $500 \mathrm{pb}^{-1}$, an accuracy of $\pm 60 \mathrm{MeV}$, largely dominated by statistics. A recent paper (Sjostrand 93) claims that the main systematic error $(\sim 40 \mathrm{MeV})$ could actually result from QCD effects leading to 'cross-talk' between the two Ws: this deserves further investigation.

Figure 3 shows the impact of such a measurement on the SM test. It is however obvious that the knowledge of the top mass is also required.

Only a LC may, eventually, do better for the $m_{W}$ measurement, provided systematic errors are well mastered.

\subsubsection{Combinations of observables}

Actually the goal of accurate measurements is to identify the potential sources of the discrepancies, if any, and to distinguish genuine new effects from effects which are due for instance to the top. To do so, one was led, by combining basic observables, to build new sets of variables, reacting specifically and in a complementary way to the various sources.

Examples are:

i) The $S$ and $T$ variables of Peskin 90: $T$ parametrizes isospin-breaking loop effects. $S$ is a measure of the potential existence of new fermion doublets.

ii) The $\varepsilon_{i}$ variables of Altarelli 90 .

iii) The variables of Blondel 91 used in Fig. 5.

The last two sets of variables have close analogies with the first one.

\subsection{The Top-Mass Determination}

According to the indirect mass determination quoted above, the top should be observed soon at the Fermilab Collider ${ }^{\dagger}$. The promises of top physics at future machines are high (Peskin 91, Zerwas 91, Kuhn 93, Froidevaux 93). But first the measurement of its mass will allow the main unknown parameter in the SM to be fixed, and sensitivity to smaller effects to be obtained, which could be due to the Higgs or to new physics. What

\footnotetext{
t) At the time of printing a press conference in Fermilab announced evidence, from the CDF experiment, for a top at $m_{t}=174 \pm 17 \mathrm{GeV}$, a value in excellent agreement with the LEP indirect measurement.
} 
accuracy can we expect for $m_{t}$ and what impact will this make on the search for new physics?

Once the top is discovered, hadron machines will provide its mass to an accuracy of about $\pm 5 \mathrm{GeV}$, perhaps slightly better.

It will probably be necessary to wait for an $\mathrm{e}^{+} \mathrm{e}^{-}$linear collider to get $m_{t}$ with an accuracy of less than $1 \mathrm{GeV}$. The behaviour of the $t \bar{t}$ system near threshold is peculiar and has been well studied (Strassler 90, Bagliesi 92, Fujii 93). With such a heavy top, no toponium spectroscopy is foreseen. The rise of the $t \bar{t}$ cross-section at threshold (Fig. 4) is described by a complicated function, the main variables being $m_{t}$ and $\alpha_{s}$, which are strongly correlated. Adding, as a second measurement, the momentum spectrum of the produced top, which has a different correlation pattern, $m_{t}$ and $\alpha_{s}$ can be obtained independently, and with great accuracy. Typically, $\Delta m_{t}=0.5 \mathrm{GeV}$ for the range of $m_{t}$ considered. It is often said that such a step in accuracy for $m_{t}$ does not help much in the overall testing of the SM. If, for instance, the sensitivity to the Higgs mass is considered, the statement is correct, as long as other measurements stay at the level of accuracy provided by the LEP/SLC era.

\subsection{The Next Round of Accurate Measurements?}

However, improvements are foreseeable in the future. A test in depth of the SM is being performed by confronting several accurate measurements. Figure 5 (Blondel 91) shows that the sensitivity to the Higgs mass is optimized by using, for instance, the top mass measurement, and an excellent determination of $\sin ^{2} \theta_{W}$. The width of the oblique band corresponds in the figure to $\Delta \sin ^{2} \theta_{W}= \pm 10^{-4}$, an accuracy three times better than that expected from LEP/SLC programmes.

A breakthrough in the accuracy of $\sin ^{2} \theta_{W}$ will require:

i) A much better measurement of $A_{L R}$ on the $Z^{0}$ : this could be obtained if one day high luminosity and large polarization coexist in LEP. It could also come from a polarized $Z^{0}$ factory (Omori 93), the limit on the precision being probably set there by the limit on the knowledge of the electron polarization.

ii) A more accurate determination of $\alpha\left(Z^{0}\right)$. It is unfortunate that the extreme accuracy of $\alpha$ at the Thomson limit is of no use for testing the SM: what matters is the precision with which the running of $\alpha$, from $\sqrt{s}=0$ to $\sqrt{s}=m_{Z}$, is known. This is governed by the knowledge of the vacuum polarization effects, which in turn depend on the quality of the measurement of $e^{+} e^{-} \rightarrow$ hadrons between threshold and $\sim 10 \mathrm{GeV}$ (Alexander 88). We can note that the exploitation of the next round of the $\mathrm{g}-2$ experiment is also bound up with such an improvement, but in a region more concentrated near threshold (Kinoshita 93).

If both conditions are satisfied, and $\Delta \sin ^{2} \theta_{W}=10^{-4}$ is reached, then it is possible to see from Fig. 6 (JLC 92) the kind of improvement on the sensitivity to the Higgs mass (considered here merely as an estimator of quality) provided by a better measurement of $m_{t}$.

\section{Direct Searches: the methods}

\subsection{Searches for peaks in mass distribution}

This is clearly the gold-plated way to discover a new particle, as shown by past successes.

$\mathrm{e}^{+} \mathrm{e}^{-}$machines will produce $1^{--}$objects in the $\mathrm{s}$ channel directly: the visibility of narrow structures is therefore governed by the resolution in centre-of-mass energy. 
For unknown narrow objects the main problem is obviously to find them. The radiative tail, by providing luminosity at lower energies, may eventually offer a useful autoscan situation, but will usually be a nuisance. It is probably safer to consider $\mathrm{e}^{+} \mathrm{e}^{-}$machines as those where studies rather than discoveries of such objects are made, unless the narrow resonance is followed by a continuum at higher $\sqrt{s}$, as in the case of heavy flavours, so that a few-step scanning procedure is possible.

For other quantum numbers, inclusive production at $\mathrm{e}^{+} \mathrm{e}^{-}$and hadron machines have to be looked for. Searches using dilepton or multilepton final states are the most promising with any machine, but to increase statistics, the use of missing quadrivectors and jets for building up mass spectra is very desirable: this should be easier to achieve at $\mathrm{e}^{+} \mathrm{e}^{-}$machines. The key factor of such studies is obviously the quality of the mass resolution.

Clearly, for reasonably narrow resonances and simple modes, the large domain of mass offered by future hadron colliders opens a wide set of possibilities.

\subsection{Searches for anomalies in kinematical distributions}

The presence of non-standard objects will modify the shape of kinematical distributions, compared to the SM expectation. In hadron machines one is limited to the distribution of transverse quantities: at $\mathrm{e}^{+} \mathrm{e}^{-}$machines, for annihilation channels, all distributions can be exploited provided bremsstrahlung and beamstrahlung are well understood (Chen $93)$.

Even within the SM, the correct prediction of such distributions may not be trivial because of radiative (QED and QCD) effects. Finally, instrumental distortions must be avoided or understood.

A special case, more akin to indirect searches, is the search for an anomalous rate of a given final state: here the mastering of the normalization is essential, a strong point of $\mathrm{e}^{+} \mathrm{e}^{-}$machines (Frary 93).

Figure 7 shows how the existence of heavy quarks at LEP I was ruled out by inspecting distributions of well-chosen quantities in the $Z^{0}$ final state. Many such examples can be found in the literature (Giacomelli 93), and the results are given in Table 1.

The most celebrated and promising of these variables are missing energy and momentum (or the transverse version of these quantities). Missing quadrimomentum is expected from escaping neutrinos - an irreducible source-and from lost particles-a source that can be decreased by improving the hermeticity of the detector. The interest in looking for such extra sources is obviously connected to supersymmetry as we will see in Section 9.

\subsection{Use of heavy-flavour tagging}

This is mostly related to the properties of the top (expected to decay to bW) and the Higgses (expected to couple to the highest available mass objects, namely the b quark and $\tau$ lepton for the mass region presently under investigation).

It has now been demonstrated that heavy-flavour tagging is performed best through the presence of offsets or secondary vertices revealed by a microvertex detector: other features (leptons, strangeness, event shape) only bring complementary, albeit useful, information.

When Ws are a source of background, b-tagging is particularly efficient since these bosons do not produce b's. 
Depending on the machine, various considerations of backgrounds and acceptances determine the efficiencies of heavy-flavour tagging. Achieved and expected figures at LEP are shown in Fig. 8 (Berggren 93), as an example.

\section{$6 \quad$ What to Search for? A Few Scenarios}

As we said previously, within the SM, and assuming that the top and $\nu_{\tau}$ appear as expected, the main question is about the electroweak symmetry breaking (EWSB) and the scalar sector. The SM postulates that the Higgs mechanism (Appendix 1) is responsible for EWSB and traditionally assumes that, after symmetry breaking, a scalar particle, the SM Higgs boson $H_{S M}^{0}$, is left, waiting to be discovered. This is the main goal of present and future programmes, and we will describe these searches in Section 7.

However, as we have already mentioned, the SM Higgs sector has defects, suffering in particular from the scalar mass instability in the presence of a high scale. For such reasons the credibility of the standard version has decreased in favour of others considered as more sound ones.

The first is supersymmetry, which we shall discuss in Section 9. Supersymmetry (Appendix 2) allows stabilization of the scalar system by compensating quadratic divergences. It gives a new status to Higgs bosons by relating their mass to the one of gauge bosons. Supersymmetry predicts the existence of many new particles, superpartners of the usual ones, and of a rich spectrum of Higgs particles, which must, at least some of them, be quite light. Supersymmetry, if it represents the truth and if its realization is not too different from our expectations, should offer clear experimental signatures, and may provide a new golden age of particle physics.

There are however other scenarios which can eventually lead to a more dull situation. What about a very heavy Higgs? One can think of letting the quadratic divergences operate: the values of the coupling $\lambda$ introduced in Appendix 1 and $M_{H}$ increase. When $\lambda \sim 1$, a strongly-interacting sector appears. The width of the Higgs boson gets larger and larger, and for $M_{H}>1 \mathrm{TeV}$ the notion of a particle disappears. One then makes the link with a case where there is no Higgs, but instead a strong interaction in the scalar sector, concerning the longitudinal components of intermediate vector bosons (IVB), as we will discuss in Section 8.

A similar situation occurs in models which a priori do without the Higgs mechanism. The $W$ and $Z$ masses could then be due to a dynamical symmetry breaking, like in technicolour, or to a composite nature of the intermediate bosons.

Technicolour (TC) keeps the frame of gauge theories: it uses a strong extension of the SM group to a group $\mathrm{G}_{T C}$ [for instance $\mathrm{SU}(4)$ ] which corresponds to a confining situation, like in QCD, with a scale $\wedge_{T C}$. One postulates the existence of new fermions, the technifermions, whose fundamental states are in the fundamental representation of $\mathrm{G}_{T C}$. These fermions can form condensates, in particular pseudoscalars: a triplet of these can be used to provide the longitudinal states $\mathrm{W}_{L}$ and $\mathrm{Z}_{L}$, and give the masses.

Another mechanism of dynamical symmetry breaking could be associated with a condensate of top quarks (Bardeen 90). The predicted top mass as a function of the scale of symmetry breaking seems to be a bit too large $(\gtrsim 200 \mathrm{GeV})$ in the minimal $t \bar{t}$ condensation model: it is however safe to consider that this possibility is still open.

Finally, more radical solutions exist which assume the existence of an underlying level of subconstituents, and strongly modify the structure of the SM. The weak interaction is then described as a residual manifestation of this new interaction. Most of the SM properties can be recovered, except that there is no Higgs. On the other hand, 
new bound states may exist, with masses of the order of the compositeness scale (see Section 13).

\section{$7 \quad$ The Standard Model Higgs}

Ignoring for a while the caveat presented above, the first task is to search for the SM Higgs. The description of such a programme has been given so many times (Gross 92, Grivaz 92) that I will limit myself to a brief status report of the present situation, and a survey of future prospects.

\subsection{Production and Decay}

The features of Higgs boson production and decay (Dawson 89) are dominated by its property of coupling preferentially with the highest-mass objects available.

In $\mathrm{e}^{+} \mathrm{e}^{-}$collisions the SM Higgs production occurs through bremsstrahlung and fusion processes (Fig. 9), the latter dominating at high energies. In hadronic collisions, the evolution of $\mathrm{gg}$ and $q q$ processes when $m_{H} / \sqrt{s}$ increases, is shown by Fig. 10 .

The expected decay modes of the SM Higgs boson as a function of its mass are well known : dominance of $b \bar{b}$ up to $\sim 140 \mathrm{GeV}$, and then of intermediate boson pairs (Fig. 11). The $2 \gamma$ decay mode, vital for the exploration of the intermediate mass region (90 to $140 \mathrm{GeV}$ ) at $\mathrm{HC}$, occurs through loop diagrams and has a small branching ratio $\left(\sim 10^{-3}\right)$.

\subsection{Present Status at LEP I}

The only useful channel at LEP energies is the bremsstrahlung ee $\rightarrow$ ZH, with either a virtual Z (LEP I) or a real one (LEP200). LEP I has rapidly excluded the existence of a light Higgs: in particular, peculiar topologies expected from its finite lifetime at very low mass have been thoroughly exploited (Grivaz 92).

For the Higgs masses at present under consideration at LEP, $H^{0}$ decays mostly into $\mathrm{b} \bar{b}$ : this explains the potential importance of b-tagging, which however has not yet been greatly exploited. The $Z^{0}$ can decay to $\nu \bar{\nu}, \ell^{+} \ell^{-}$, or $q \bar{q}$. This defines the three main final states to explore:
a) $\nu \bar{\nu} \quad b \bar{b}$
b) $\ell^{+} \ell^{-} b \bar{b}$
c) $q \bar{q} \quad b \bar{b}$

At LEP I (c) is swamped by QCD 4-jet background. (b) is clean, but has a low rate at high Higgs mass, and an irreducible background source of 4 fermions (Glover 89). As illustrated in Fig. 12, (a) is the most useful channel, and has provided the present limit: $m_{H}>63.5 \mathrm{GeV}$ (Rubbia 93). Table 2 lists the few potential Higgs candidates left. There is no mass accumulation and none appears in the vicinity of the mass limit, so the usual CL limits with zero background can be applied.

According to preliminary results including 93 data, ALEPH, by itself, now provides a limit slightly beyond $60 \mathrm{GeV}$.

The mode $\mathrm{Z} \rightarrow \mathrm{H} \gamma$, whose rate overtakes the bremsstrahlung one for $m_{H}>60 \mathrm{GeV}$, is of no use because of an overwhelming background of ee $\rightarrow q \bar{q} \gamma$. At present a factor $\gtrsim 100$ is missing in order to have access to this channel (Tabarelli 93). b-tagging and a better mass resolution may bring an order of magnitude improvement. This is not enough for getting sensitivity to the SM Higgs, but it may be interesting to test non-standard possibilities (Dusedau 86, Hagiwara 93). 


\section{Table 2}

Higgs candidates with masses $>25 \mathrm{GeV}$ from the four collaborations [from Rubbia 93]. For the signal the rates of eeqq, $\mu \mu \mathrm{qq}, \nu \nu \mathrm{qq}$ should be proportional to $1,1,6$.

\begin{tabular}{|c|c|c|c|}
\hline Expt. & Signal & $M(\mathrm{GeV})$ & Background \\
\hline \multirow[t]{3}{*}{ ALEPH } & eeqq & none & $\approx 1.5$ \\
\hline & $\mu \mu \mathrm{qq}$ & none & \\
\hline & $\nu \nu \mathrm{qq}$ & none & $\approx 0.24$ \\
\hline \multirow{4}{*}{$\begin{array}{l}\text { DELPHI } \\
\text { (prel.) }\end{array}$} & eeqq & $35.4 \pm 5.0$ & $1.0 \pm 1.0$ \\
\hline & eeqq & $38.2 \pm 5.0$ & \\
\hline & eeqq & $17-40$ & \\
\hline & $\mu \mu \mathrm{qq}$ & $28.9 \pm 5.0$ & $1.9 \pm 0.4$ \\
\hline \multirow{4}{*}{ L3 } & $\nu \nu \mathrm{qq}$ & none & $25 \perp 04$ \\
\hline & $\begin{array}{l}\text { eequ } \\
\text { eeg }\end{array}$ & $67.6 \pm 0.7$ & $2.0 工 0.4$ \\
\hline & $\mu \mu \mathrm{qq}$ & $70.4 \pm 0.7$ & $1.2 \pm 0.2$ \\
\hline & $\nu \nu \mathrm{qq}$ & none & \\
\hline \multirow{3}{*}{$\begin{array}{l}\text { OPAL } \\
\text { (prel.) }\end{array}$} & eeqq & none & $1.2 \pm 0.4$ \\
\hline & $\mu \mu \mathrm{qq}$ & none & \\
\hline & $\mu \mu \mathrm{qq}$ & $25.1 \pm 3.0$ & $0.15 \pm 0.15$ \\
\hline
\end{tabular}

\subsection{Prospects at LEP200}

These was studied in Aachen 87 and more recently in a LEPC Workshop (Treille $93)$.

For a given Higgs mass, the cross-section for $\mathrm{e}^{+} \mathrm{e}^{-} \rightarrow H^{0} Z^{0}$ at LEP200 has the energy profile shown in Fig. 13: a steep rise at threshold, a maximum value reached for $\sqrt{s} \simeq m_{H}+m_{Z}+\sim 10 \mathrm{GeV}$, and a slow decrease when $\sqrt{s}$ increases. As a function of $m_{H}$, the maximum value of the cross-section is also slowly decreasing.

The search channels are the same as at LEP I, with a real $\mathrm{Z}$ in the final state. The backgrounds channels are shown in Fig. 14.

A complete tree-level calculation of the $\nu \bar{\nu} b \bar{b}$ final state in the upper energy range of LEP200 can be found in Dubinin 93, Boos 93.

The impact of b-tagging on the significance of the signal is obvious from Fig. 15 (Alcaraz 93), and becomes vital when $m_{H} \sim m_{Z^{0}}$. Hermeticity of the detector for the $\mathrm{H} \nu \nu$ channel is another important requirement.

Figure 16 shows the discovery potential at LEP200 (Janot 92). We can summarize the results by quoting a mass reach of $m_{H} \simeq \sqrt{s}-100 \mathrm{GeV}$ for $\sim 250 \mathrm{pb}^{-1}$, obviously reflecting the properties of the cross-section described above.

\subsection{Prospects at Linear Colliders}

The problem is quite similar to the one at LEP200 as long as the bremsstrahlung process dominates: in particular b-tagging will be very useful. It should however be remembered that for a given Higgs mass the Higgs cross-section slowly decreases, whilst various fusion backgrounds rapidly increase with $\sqrt{s}$ (Fig. 14): the optimal mode for Higgs study, once it is discovered, is therefore to bring the energy of the machine down to $\sim m_{H}+110 \mathrm{GeV}$.

For $m_{H} \sim 100-150 \mathrm{GeV}$, at the NLC $(\sqrt{s} \sim 500 \mathrm{GeV})$ the fusion mechanism takes over. This machine will allow the discovery of a Higgs boson up to $\sim 350 \mathrm{GeV}$, but a 1.5 TeV machine is needed to cover the full mass domain. Various studies (Janot 93, 
Watanabe 93) have demonstrated that the visibility of a Higgs boson at an LC is guaranteed when the c.m. energy is sufficient.

As previously mentioned, an $\mathrm{e}^{+} \mathrm{e}^{-}$(or $\mathrm{e}^{-} \mathrm{e}^{-}$) collider can, in principle, be turned into a $\gamma \gamma$ collider: this would be an ideal machine to study (but not to discover) the Higgs boson (Richard 91, Brodsky 93, Telnov 93). It is produced there through graphs containing loops in which all fermions circulate, the heaviest ones dominating the cross-section. It is thus a way to count the number of heavy fermions. At low $\sqrt{s}(\sim 100 \mathrm{GeV})$ the $\gamma \gamma$ collider should be well suited to study the intermediate mass Higgs boson observed in the $\mathrm{b} \bar{b}$ mode. However at higher $\sqrt{s}$, when the Higgs boson is detected in Z pairs (actually $\mathrm{Z}_{L} \mathrm{Z}_{L}$ ), the loops involving the $\mathrm{W}$ introduce a background which becomes lethal for $m_{H}$ $>300 \mathrm{GeV}$, unless longitudinal and transverse $\mathrm{Z}$ modes can be disentangled by a full final-state analysis (Jikia 93). Further studies are needed.

\subsection{Prospects at the LHC/SSC}

Future large hadron colliders have a large potential for the exploration of the Higgs sector (Virdee 93, Pauss 94).

The production cross-section shown in Fig. 10 is relatively comfortable up to $\mathrm{M}_{H} \sim$ $800 \mathrm{GeV}$.

When the Higgs boson decays substantially into a pair of $Z \mathrm{~s}$ and is abundantly produced, namely for $140 \lesssim m_{H} \lesssim 800 \mathrm{GeV}$, the search, through four lepton final states, is relatively straightforward.

On the other hand, the extreme regions below $\sim 140 \mathrm{GeV}$ (a) and above $\sim 800 \mathrm{GeV}$ (b) are certainly quite difficult to explore. In (a) the dominant $b \bar{b}$ decay mode is out of reach because of background: one must rely on the $\mathrm{H} \rightarrow \gamma \gamma$ mode in spite of its small branching ratio. With an outstanding electromagnetic calorimeter, retaining its quality at full luminosity, the signal should be visible over the irreducible $2 \gamma$ background spectrum (Abe 93b): this assumes that the reducible background from $\pi^{0 \prime}$ s or jets mimicking a photon, can be mastered, as well as the background from $Z^{0} \rightarrow e^{+} e^{-}$, with the e confused with a $\gamma$, for masses around $90 \mathrm{GeV}$.

Above $\sim 800 \mathrm{GeV}$, various tricks as those described in the case of a strongly interacting sector (central jet veto, forward jet tag) have to be used.

If reality conforms with Monte Carlo expectations, the $\mathrm{HC}$, with several years at full luminosity, should solve the SM Higgs problem.

Figure 17 summarizes the overall prospects for Higgs search.

\section{The Strongly Interacting Scenario}

\subsection{Generalities}

In such scenarios (Chanowitz 92, Bernreuther 93) symmetry breaking leads to a strong interaction of the longitudinal component of IVB. There are no light narrow bosons to look for: the signal is a departure from the normal IVB interaction.

This strong interaction may or may not be resonant. In the latter case it nevertheless obeys low-energy theorems (LET), dictating the behaviour of the boson-boson scattering amplitudes with energy. Resonances may occur in different spin-isospin states.

The various models either stretch the situation of the SM up to large $m_{H}$ (Higgs-like models), or mimick the QCD picture with vector resonances with $\mathrm{I}=1$ ( $\rho$-like) or $\mathrm{I}=0$ ( $\omega$-like). Technicolour or the BESS model (Casalbuoni 85, Casalbuoni 94) belong to the latter category. A more general approach (Dobado 90) can generate both aspects. 
As a preliminary question, we could ask ourselves whether it is possible, from accurate measurements, to tell in advance if nature has chosen the strongly interacting scenario. This has been addressed for instance in Gaillard 92, Layssac 93. At an $\mathrm{e}^{+} \mathrm{e}^{-}$ machine, a resonance can indeed be felt at a somewhat lower c.m. energy, from asymmetry measurements. For instance, LEP200 would allow the presence of a resonance of 300$450 \mathrm{GeV}$ to be seen. This statement is however model dependent, and somewhat disappointing since it is necessary to come 'close' in energy to the new physics to notice it.

In the case of technicolour, which postulates a number of new technicolours and another number of new technidoublets, a positive $S$ variable (see Section 4.3.4) is expected, whilst experiment favours a small and negative value. However, it may be possible, according to some authors (Luty 93), to save the situation in more complex technicolour models. Apart from such indirect hints it is clear that the answer belongs to machines with the largest c.m. energy.

\subsection{Prospects at Linear Colliders}

At these machines, two possibilities are offered (Fig. 18): ee $\rightarrow$ WW accurate measurement, and $\mathrm{W}_{L} \mathrm{~W}_{L}$ fusion.

The latter is first characterized by its differential luminosity curve (Hikasa 91, Hagiwara 91): other types of collisions, $\gamma \gamma, W_{T} W_{T}$, by far dominate the interesting one, $W_{L} W_{L}$ (Fig. 19a). The relevant cross-section is small, and even tiny in the nonresonant case (Fig. 19b). A possible way to exhibit a strong rescattering in the $W_{L} W_{L}$ channel is to exploit the corresponding phase shift, and observe an interference between $\mathrm{W}_{L} \mathrm{~W}_{L}$ and $\mathrm{W}_{T} \mathrm{~W}_{T}$ amplitudes. This seems however beyond realistic possibilities (Hikasa 91).

The other possibility is to perform an accurate study of the $\mathrm{e}^{+} \mathrm{e}^{-} \rightarrow \mathrm{W}^{+} \mathrm{W}^{-}$channel, measuring completely the final state. This has been simulated for instance in Barklow 91. Considering the resonant case, let us assume the existence of a techni-rho of mass $m_{\rho}$ : this resonance is described by a rescattering function $F$ corresponding to an Argand loop which depends on $m_{\rho}$ (Fig. 20). From a global likelihood fit to the final-state observables, one has determined (Barklow 91, Barklow 93) the degree of observability of such a resonance, as a function of its mass, for various conditions of energy and luminosity.

The result shown in Fig. 21 leads us to conclude that, for the illustrative technirho mass of $1.7 \mathrm{TeV}$, an NLC is marginal, and it will be necessary to get either much more integrated luminosity or a higher c.m. to observe the effect clearly. As for the nonresonant case, it has been shown in the same work that both a very high c.m. energy and a very high luminosity-beyond our 'reasonable' assumption of Section 2-are required to achieve the sensitivity needed.

No doubt a strongly-interacting scenario may be a difficult case to identify at an LC: to have a chance to do so, a machine well beyond the NLC, in energy and/or luminosity, will be needed. The ability to perform accurate and clear measurements is the best promise of such a type of machine, and this quality should be preserved when increasing the energy and luminosity.

\subsection{Prospects at Future Hadron Colliders}

It has often been stated that HCs (the SSC at $10^{33}$, and the LHC at $10^{34}$ ) offer a 'no-lose' situation. This means that whatever is the version of electroweak symmetry breaking, they will observe and identify it. In particular this implies that they will be able 
to do so for any strongly-interacting scenario. The processes which potentially allow the achievement of this goal are those leading to pairs of bosons.

Several versions of such estimates have been given in the literature (Chanowitz 92, Bernreuther 93). A recent paper (Bagger 93) claims that by restricting the analysis to the gold-plated modes where IVB decay leptonically, and by using several tricks, such as forward jet tagging and central jet veto to decrease the backgrounds (Fig. 22), it is possible to arrive at a manageable situation.

Figure 23 shows various plots of quantities built from boson pair leptonic decays, such as for instance the dilepton mass. They indicate a difference in shape between the background, and an eventual signal, even in the case of a non-resonant behaviour.

However, one must realize how limited the statistics are in such a case: no doubt several years of running at full luminosity, and a good control of the detection efficiency along the spectra will be needed to give a meaningful answer.

See Bagger 93 for a more complete discussion of these arguments.

\section{$9 \quad$ Supersymmetry}

\subsection{Generalities}

The idea of supersymmetry is now well known (Zwirner 93, Fayet 93). We will just insist on phenomenological consequences.

In its simplest version the Higgs sector of SUSY is very interesting and constrained. From the two complex doublets needed for electroweak breaking, after the Higgs mechanism has operated, five particles are left: two scalars, $h^{0}$ and $H^{0}$, a pseudoscalar $A^{0}$, and two charged bosons $H^{ \pm}$. Of these, at least $h^{0}$ has to be very light (see later).

Actually in the most simple version of supersymmetry, the Minimum Standard Supersymmetric Model (MSSM), the number of free parameters is small and, in the Higgs sector, quantitative estimates are possible. Table 3 gives the relevant parameters for each of the MSSM sectors. It also recalls the expected relationship between the masses of various objects. These relations result from the assumed simplicity at high scale, modified by an evolution described by renormalization group equations. An important prediction is, for instance, the expected ratio of the gluino mass and the lightest supersymmetric particle (LSP) or chargino masses.

\section{Table 3}

Set of MSSM parameters describing its various sectors and expected relations between masses $\left(m_{1 / 2} \equiv \mathrm{M}\right.$ in the text) [from Amaldi 92)]. 


\subsection{What can we tell in advance about supersymmetry?}

Supersymmetry is an example of a theory for which the argumentation about accurate measurements developed in Section 4 does not apply: we have here a decoupling situation (Haber 92).

\subsubsection{Modifications of the $Z^{0}$ properties}

It may be that supersymmetric objects (gauginos, etc.) exist at a quite low mass, and will even be accessible at LEP200. In such a case, the vicinity of the threshold could be felt on the $Z^{0}$ by a renormalization of its wave function, caused by virtual gauginoshiggsinos: this would lower all $\mathrm{Z}$ widths, without affecting the asymmetries, and would show up in a low value of the parameter $\varepsilon_{3}$ (Barbieri 91a).

Unfortunately the present accuracy and the expected one at the end of the LEP I phase does not leave much hope of a really convincing demonstration. A next round of accurate measurements (see Section 4) would probably provide such an answer. But given the proximity of the new objects, this is an example of a situation where it is more rewarding to invest in c.m. energy, and observe (or exclude) these particles.

\subsection{D $b \rightarrow s \gamma$}

Radiative b decays proceed through loop (penguin) diagrams (Fig. 24), and their rate is enhanced by gluon exchange (Bertolini 91). In the case of SUSY, new objects $\left(H^{ \pm}\right.$, but also charginos, etc.) intervene in the loops, besides the W: a complete calculation including all effects has to be made in order to determine the actual overall effect. A theorem (Ferrara 74) states that, in the limit of exact supersymmetry, the rate of $b \rightarrow$ $\mathrm{s} \gamma$ vanishes identically because a magnetic transition is forbidden. In broken SUSY, as Fig. 25 (Barbieri 93) indicates, it is then possible to find rates below or above the SM one, depending on the location in the SUSY plane. Present measurements do not allow any clear cut conclusions to be drawn (Borzumati 93): one trend is that for low $\operatorname{tg} \beta$ the Higgs masses, except $m_{h^{0}}$ which stays small, are pushed above $\sim 200 \mathrm{GeV}$ (Bertolini 94).

However, in a two-doublet model (not SUSY), where $H^{ \pm}$is the only new particle participating in the loops, it is true that these results set a lower limit on $m_{A}$ (Hewett 93).

\subsubsection{Grand Unification(Langacker 81, de Boer 93)}

Up to now, as we said in the Introduction, an encouragement for seriously considering the supersymmetric option comes from the apparent convergence, at very high energy, of the three running coupling constants of the fundamental interactions (Amaldi 91).

Starting from their values at $\sqrt{s}=M_{Z}$, accurately measured at LEP and elsewhere, the evolution of the couplings, as shown in Fig. 26, is described by a slope: this slope is governed by the population of particles intervening in the loop diagrams (i.e. screening or antiscreening the corresponding interaction).

In the SM the slopes are such that the convergence does not happen. On the contrary, it happens for SUSY: in this case, crossing the SUSY threshold is approximated by a 'kink' whose location, common to the three curves, is a free parameter, found to be in the $(100 \mathrm{GeV}$ - few $\mathrm{TeV})$ region. In the literature, it is referred to as $\mathrm{M}_{S U S Y}$ or $\mathrm{M}_{s q}$.

This result does not provide a proof, but it would be unwise to ignore such a hint.

\section{Supersymmetry: The Higgs Sector}

The predictions for the Higgs sector in the MSSM are very striking (Zwirner 93). We can describe them approximatively with four figures (27-30), which we shall now explain. 
In this sector, essentially described by two parameters once $m_{t}$ is known and $m_{\tilde{t}}$ assumed, one chooses either $\operatorname{tg} \beta-m_{A}$ or $m_{h}-m_{A}$ (the latter being an equivalent parametrization, but for which $\operatorname{tg} \beta$ is not single valued).

At tree level the value for $m_{h^{0}}$ should be limited upwards by $m_{Z^{0}}$. Actually, radiative corrections (Okada 91, Ellis 91, Haber 91, Barbieri 91b) owing to the top and its superpartner, the stop, raise this upper limit to

$$
m_{h^{0}}^{\max } \simeq m_{Z^{0}}+\varepsilon,
$$

where $\varepsilon$ is a correction that depends greatly on $m_{t}$, and modestly on $m_{\tilde{t}}$. Figure 27 gives, for $m_{\tilde{t}}=1 \mathrm{TeV}$, the upper limit on $m_{h^{0}}$ as a function of $m_{t}$ and $\operatorname{tg} \beta$. For $m_{t}=160 \mathrm{GeV}$ this limit is $120 \mathrm{GeV}$. Two very recent calculations of two-loop radiative corrections have appeared (Hempfling 93, Kodaira 93). Although the values of the mass limit evolve in different ways, both computations seem to lead to about the same numerical value. For $m_{\tilde{t}}=1 \mathrm{TeV}$, and $m_{t}=150 \mathrm{GeV}(170 \mathrm{GeV})$ the mass limit is (Fig. 31)

$$
m_{h^{0}}<108 \mathrm{GeV}(118 \mathrm{GeV}),
$$

values which are somewhat lower than the one quoted previously.

Figure 28 shows the masses of the Higgs bosons as a function of $m_{A}$ for three values of $\operatorname{tg} \beta$ : it can be seen that when $m_{A}$ grows, all the masses grow as well (the bosons become approximately mass degenerate with $\mathrm{A}$ ), except $m_{h^{0}}$ which stays small and constant.

Figure 29 gives, for $m_{t}=160 \mathrm{GeV}$ and $m_{\tilde{t}}=1 \mathrm{TeV}$, the isomass curves for $h^{0}$ in the $\operatorname{tg} \beta-m_{A}$ plane. It can be seen that in the upper right part of the plot the mass is nearly stationary.

Furthermore, at the right of the $\operatorname{tg} \beta-m_{A}$ plane, the rate is practically the SM one (Fig. 30). From what we said for the SM Higgs, we can understand that the $h^{0}$ discovery limit at an $\mathrm{e}^{+} \mathrm{e}^{-}$machine will also be

$$
m_{h^{0}} \simeq \sqrt{s}-100 \mathrm{GeV}
$$

corresponding approximately to an isomass curve in Fig. 29.

It can also be understood, looking at Fig. 29, that a small step in $\sqrt{s}$ can dramatically change the coverage of the $\operatorname{tg} \beta-m_{A}$ plot.

Moreover, the associated production modes $\mathrm{e}^{+} \mathrm{e}^{-} \rightarrow \mathrm{hA}, \mathrm{HA}$ and $\mathrm{e}^{+} \mathrm{e}^{-} \rightarrow \mathrm{H} Z^{0}$ should be considered as well. Their importance depends crucially on the available c.m. energy.

If, instead of the upper bound, we focus on the actual mass of the $h^{0}$ boson, we can get an idea of its range of expectation by varying the parameters of the model within the region allowed by existing experimental results: an example for such a constrained MSSM is given in Fig. 32 (de Boer 94). Other ones can be found in Olechowski 92, Kane 93.

In non-minimal versions of SUSY, various studies (Espinosa 92, Elliott 92, Ellwanger 93) have shown that the $h^{0}$ boson is still light, although firm numerical predictions are no longer available. It may also be that the $\mathrm{h}^{0}$ production and decay is not standard like anymore

In Langacker 94 the set of minimal SUSY models with Grand Unification and a desert (i.e. no new physics) up to that scale have been studied: one finds that, to get the equality of the $\tau$ and b Yukawa couplings at the GU scale, $\operatorname{tg} \beta$ is either $\sim 1$ or large. In the former case the expected $h^{0}$ mass domain is the one indicated in Fig. 33. 


\subsection{The Present Situation at LEP I}

Besides the bremsstrahlung production mode

$$
e^{+} e^{-} \rightarrow h^{0} Z^{0}
$$

the other relevant one is the associated production

$$
e^{+} e^{-} \rightarrow h^{0} A^{0}
$$

The two reactions are complementary

$$
\begin{aligned}
& \sigma(1) \sim \sin ^{2}(\alpha-\beta) \\
& \sigma(2) \sim F^{3} \cos ^{2}(\alpha-\beta),
\end{aligned}
$$

where $\alpha$ is a mixing angle, $\operatorname{tg} \beta$ is the usual ratio of vacuum expectation values, and $F^{3}$ is the p-wave phase-space factor. Reaction (1) can be studied in the same way as the $\mathrm{SM}$ one, as long as $\sin ^{2}(\alpha-\beta)$ is large enough. For reaction (2) the final states to be explored depend on the assumed decay modes of the bosons. Indeed, owing to the alreadymentioned radiative corrections, the mass hierarchy of the bosons can be modified, in such a way that eventually $h^{0} \rightarrow A^{0} A^{0}$. A complete search strategy, including all possible cases for the decays, can be found in Grivaz 92, Grivaz 93.

The search for associated production leading, for high-mass bosons, to a $4 \mathrm{~b}$ final state, was the first to benefit from a high-performance b-tagging (Berggren 93). Figure 34a presents the results of this search interpreted in the two-doublet scheme: the mass limits obtained follow closely the kinematical mass limit for the hA channel up to $m_{h} \sim 40$ $50 \mathrm{GeV}$. In the MSSM frame, the mass limits are even more restrictive: while the limit on $m_{h}$ stays close to the kinematical one $(\sim 45 \mathrm{GeV})$, the limit on $m_{A}$ gets weaker for $m_{t} \gtrsim 150 \mathrm{GeV}$ and/or for a large squark mass (Fig. 34b). All values of tg $\beta$, including $\operatorname{tg} \beta \sim 1$, are still experimentally allowed.

\subsection{Prospects at LEP200}

The considerations we made before about the impact of the available c.m. energy are quite relevant for LEP200, since its end point is not yet exactly defined. The energy planned for the first phase $(\sqrt{s}=175 \mathrm{GeV})$ would only modestly increase the range of mass already explored at LEP I. The canonical energy $(\sqrt{s}=190 \mathrm{GeV})$ would cover the $\operatorname{tg} \beta-m_{A}$ plane up to the isomass curve of $90 \mathrm{GeV}$, as confirmed by Fig. 35 obtained by a full Monte Carlo study (Janot 92): this figure distinguishes the regions where the contributions from reactions (1) and (2) are respectively dominating. A further increase of energy would extend and eventually complete the exploration of the $\operatorname{tg} \beta-m_{A}$ plot. The CM energy required to do so would be $\sqrt{s} \sim 210 \mathrm{GeV}(220 \mathrm{GeV})$ for $m_{t}<150 \mathrm{GeV}$ $(170 \mathrm{GeV}$ ). This is shown in Fig. 36 (Janot 92). The plane is covered, at a $5 \sigma$ significance level for discovery, except for a tie-shaped region at the border between the two regions where the significance is between 3 and $5 \sigma$.

If, instead of the upper bound, we consider again the actual mass of the $h^{0}$ boson, we can go back for instance to Fig. 32 to get an idea of the detectability at LEP200 as a function of $m_{t}$ (the limits of Fig. 32 are obtained at first order). Similarly Fig. 37 gives an estimate, from another version of a constrained MSSM (Kane 93), of the chance to detect the $h^{0}$ at an $\mathrm{e}^{+} \mathrm{e}^{-}$machine as a function of its centre-of-mass energy.

In non-minimal versions of SUSY such statements are no longer possible. However, it is still true that even a modest increase of $\sqrt{s}$ in the $200 \mathrm{GeV}$ region very rapidly 
multiplies the chance to find the $\mathrm{h}^{0}$ (and possibly gauginos, etc. as well), if SUSY is a reality. We presented in Fig. 33 (Langacker 94) a quite likely possibility for which the relevance of $\sqrt{s}$ at LEP200 is manifest.

\subsection{Prospects at Linear Colliders}

At an LC, even with a c.m. energy as low as $\sim 250-300 \mathrm{GeV}$, the problem of threshold energy disappears, and the $h^{0}$ will be observed if it exists and is produced at a sufficient rate (Janot 93). If it is not found the MSSM is ruled out and most of the nonminimal versions as well. If a light scalar is discovered the question is then to determine whether we are facing supersymmetry or not.

One way is to observe the partners of the $h^{0}$ through the processes quoted above. One can, for instance, look at the $\tau \tau \mathrm{q} \bar{q}$ mode: the visibility of these partners is guaranteed (Fig. 38). However, Fig. 28 reminds us of the evolution of masses with $m_{A}$ : for $m_{A} \geq \sqrt{s} / 2$ an LC will run out of c.m. energy to produce the $h^{0}$ partners.

Another way is to measure accurately the branching ratios of the $h^{0}$ : it has been shown (Hildreth 93) that up to $m_{A} \sim 350 \mathrm{GeV}$ at the NLC it is still possible, but somewhat marginally, to distinguish the MSSM from the SM. The general situation is summarized in Fig. 39 (Janot 93).

Moreover, at a LC, the visibility of the charged Higgs, $H^{ \pm}$, is guaranteed up to a mass which is close to $\sqrt{s} / 2$ (say $\geq 85 \%$ of it), whatever is the decay (Sopzcak 93).

Clearly if SUSY is the truth, an LC is an excellent machine to explore its Higgs sector.

\subsection{Prospects for the SUSY Higgs Sector at Hadron Colliders}

At an HC, in spite of the large $\sqrt{s}$ available, the situation is difficult. The only possible direct observation at low mass, as in the SM case, is $h^{0} \rightarrow \gamma \gamma$ : the experimental problem is the same, except that the branching ratio decreases when $m_{A}$ decreases (Kunszt 91). The discovery limit is the vertical line of Fig. 40. A four-lepton final state can exclude the region indicated in Fig. 40, which is however already mostly covered by LEP200. The other possibility is to observe a violation of lepton universality caused by

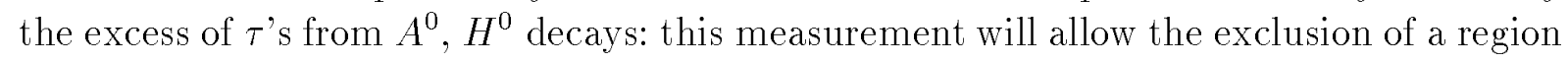
at the top of Fig. 40. Details are given in Virdee 93, Pauss 94.

\section{Supersymmetry: Other Supersymmetric Particles}

\subsection{Generalities}

Apart from the requirement of a relative lightness $(\lesssim 1 \mathrm{TeV})$ and considerations of naturalness (Barbieri 89), there are no firm predictions about the masses of supersymmetric particles other than the lightest Higgs boson. It is usual to expect that gauginos - neutralinos and charginos - may be the 'nearest' SUSY particles. Constrained models generally find this as well (Kane 93).

The other general remark is that hadron machines are the right ones for stronglyinteracting particles (gluinos, squarks), whilst electron machines are best suited for weaklyinteracting ones (sleptons, gauginos). The expected mass relationships between gluinos and gauginos actually makes the reach of both types of machines very similar in this specific case. 
A particularly interesting object is the lightest supersymmetric particle (LSP). In the case of R-parity conservation it is stable. It is therefore the source of the most conspicuous signature expected for SUSY: missing energy and momentum. It is also an outstanding candidate for dark matter. If the LSP, denoted here by $\chi^{0}$, is the lightest gaugino, the relationship already quoted between gluino and gaugino masses implies that both types of searches are relevant to the LSP problem.

In the case of R-parity violation, the situation is very different since the LSP may be unstable.

\subsection{Gauginos}

The phenomenology of neutralinos and charginos is generally described as a function of the MSSM parameters (Appendix 2). A usual representation of the available parameter space is presented in Fig. 41, in the plane $\mathrm{M}-\mu$, for a given value of $\operatorname{tg} \beta$. The vertical scale can be interpreted in terms of the common gaugino mass at a high scale, M, or, equivalently of the LSP or gluino masses, linked by the relations of Table 3.

\subsubsection{Charginos}

Charginos $\chi_{1}^{ \pm}, \chi_{2}^{ \pm}$are the mass eigenstates corresponding to the linear combinations of winos and charged Higgsinos.

i) The present situation at LEP I (Grivaz 91)

Charginos have been searched for via their pair production and decays

$$
\begin{gathered}
\mathrm{e}^{+} \mathrm{e}^{-} \rightarrow Z^{0} \rightarrow \chi^{+} \chi^{-}, \\
\chi^{ \pm} \rightarrow W^{+} \chi^{0} \rightarrow \mathrm{q} \overline{q^{\prime}} \chi^{0}, \ell \nu \chi^{0} .
\end{gathered}
$$

Within the MSSM, the charginos would be abundantly produced at the $Z^{0}$, with rates depending on their composition: indirect searches (from the $Z$ line shape) have been sufficient to exclude their existence up to the kinematical limit, or close to it, under this assumption (Table 1 ).

From direct searches, LEP I, benefiting from the energy scan, has ruled out masses even slighty higher than $m_{Z} / 2$.

ii) LEP200 (Coignet 93)

The diagrams leading to chargino production (Fig. 42) may interfere destructively and this can substantially decrease the cross-section (Fig. 43). The chargino decay pattern depends on whether the sneutrino mass is larger or smaller than the chargino mass. Besides SUSY parameters, both the luminosity and the c.m. energy are important to assess the actual reach: in brief one can say that at LEP200 chargino masses up to $\sim 80 \mathrm{GeV}$ will be explored.

iii) Linear colliders

At these machines the same kind of searches will continue. The implications for the MSSM are summarized in Fig. 41 which illustrates the gain obtained by an LC. The chargino searches will be less efficient if $\Delta m=m_{\chi^{+}}-m_{\chi^{0}}$ becomes smaller: beyond the curve c of Fig. 41, corresponding to $\Delta m=20 \mathrm{GeV} / c^{2}$, it is unlikely that a valid exploration can be performed.

iv) Hadron colliders

Through the search of trilepton events, resulting from pair production and cascading of charginos and neutralinos, the CDF experiment has given a lower limit of $\sim 47 \mathrm{GeV}$ for its mass, valid in a subset of the parameter space; this result is equivalent to the exclusion of a gluino below $\sim 175 \mathrm{GeV}$ (Fig. 44). The Tevatron, with 
its foreseen improvements (main injector), will explore a substantially higher mass domain. At the LHC, for even higher masses, chargino searching will be a difficult enterprise: the possibility of seeing a signal will rely on a drastic reduction of the dominant $t \bar{t}$ background. However their exclusion will follow the exclusion of gluinos.

\subsubsection{Neutralinos}

Neutralinos, denoted by $\chi^{0}, \chi^{0 \prime}, \chi^{0 \prime \prime}, \chi^{0 \prime \prime \prime}$, in order of increasing mass, are linear combinations of the photino, the zino, and the higgsino (Appendix 2). Whilst $Z^{0} \rightarrow$ $\chi^{0} \chi^{0}$ is invisible, $Z^{0} \rightarrow \chi^{0} \chi^{0 \prime}$ can be searched for in the $\chi^{0} \chi^{0} f \bar{f}$ and $\chi^{0} \chi^{0} \gamma$ final states. The negative results obtained can be presented either in a model-independent way, by a bidimensional plot of $m_{\chi}$ versus $m_{\chi^{\prime}}$, or within the frame of the MSSM, as exclusion contours in the $(\mathrm{M}-\mu)$ plane (Giacomelli 93).

Concerning the coverage of this plane, neutralino searching does not bring any substantial improvement compared to chargino searching.

The main concern with neutralino searching is the mass limit that can be set on the LSP: the result so far is that at LEP, for small $\operatorname{tg} \beta \leq 2$, no mass limit has been set because in these conditions the branching ratio of the $Z^{0}$ to neutralinos fades away. This is illustrated by Fig. 45 which gives a subset of the LEP results (Grivaz 93).

\subsubsection{The LSP as a dark matter candidate}

It is a well known problem that most of the mass of the universe seems to be of an unknown nature. At least two main types of dark matter-hot and cold-are considered (Turner 93, Ellis 93a). Recent microlensing experiments (Aubourg 93, Alcock 93) may have shown that dark stars (brown dwarves) contribute to part of the baryonic cold dark matter (CDM). However, the LSP is still one of the favourite candidates for non-baryonic CDM.

High-energy accelerators will explore larger and larger masses: $\sim 20 \mathrm{GeV}$ for LEP I with the caveat just indicated, up to $\sim 80 \mathrm{GeV}$ for LEP II. Meanwhile nonaccelerator methods, based on the detection of recoils induced by relic LSP's trapped in our galaxy (Sadoulet 92, Pretzl 93), will progress from very high masses 'downwards'. An assessment of the actual potential of theses methods can be found in Drees 94. Hopefully the two fields will some day have explored the full domain of possibilities.

In parallel new experiments on neutrino oscillations, in particular those presently starting (Chorus, Nomad), will check the idea that a non-zero mass of at least one neutrino species could also contribute to explain the missing mass mystery.

\subsubsection{Gluinos}

This is the domain of HCs. The production cross-section is comfortably large. The simplest decay mode to be expected is $\widetilde{g} \rightarrow \mathrm{q} \bar{q}+\chi^{0}$. The actual ones are however subject to some ambiguity concerning the importance of cascading, and therefore of the amount of missing energy and momentum.

Present results from the Fermilab Collider are given in Fig. 46, illustrating the dependence of the limits on the assumptions about the decay modes, and the correlation of the gluino and the squark masses.

Expectations for future colliders have been the subject of many studies, as discussed in Pauss 90. Besides missing energy, other signatures such as like-sign dileptons and multi-W final states (Baer 90, Barger 92) can be exploited to prove the existence of gluinos. 
Concerning the LSP, the present limit on the gluino mass, according to the relations given above between masses, fills the hole left by $\mathrm{e}^{+} \mathrm{e}^{-}$results at small $\mathrm{tg} \beta$. It could therefore be stated that by combining LEP and Fermilab results, $m_{L S P}>20-30 \mathrm{GeV}$, whatever the value of $\operatorname{tg} \beta$.

\subsubsection{The light gluino window}

This is however not completely true: indeed a claim (controversial) is that HCs cannot exclude a very light gluino $\left(m_{\tilde{g}}<\right.$ a few $\left.\mathrm{GeV}\right)$ because they reach their limit of sensitivity to missing $E$. The 'hole' left over is shown in Fig. 47 (Albajar 87). Whether the existence of a light gluino helps to solve possible problems in other data has been much discussed (Jezabek 93, Hebbeker 93, Ellis 93b), without any significant conclusion up to now.

In the MSSM, for $\operatorname{tg} \beta<2$, no limit exists on the LSP mass. Because of this, or by considering non-MSSM variants, the possibility of the decay $\widetilde{g} \rightarrow \mathrm{q} \bar{q} \widetilde{\gamma}$, with a very light photino, is still open. No convincing cosmological argument can rule out the existence of such a photino (Valle 92, Campos 93). $\mathrm{e}^{+} \mathrm{e}^{-}$machines are a potential source of light gluinos created through diagrams leading to 4 jets (Fig. 48). The gluinos would usually be unrecognizable, except in the domains where, because of an adequate lifetime, they either have a long flight or look approximatively like a b-jet. The methods of b-tagging described before apply in the latter case. Very preliminary results from DELPHI exploit this feature. It is however doubtful that the coverage of the hole can be improved much by this method. A detailed study of 4-jet final states and their departure from QCD could lead to some progress (Schmelling 93), as well as a careful study of $\alpha_{s}$ running.

\subsection{SUSY Partners of the Basic Fermions}

\subsubsection{Squarks}

Here again HCs are the most promising (Pauss 90) since the mass reach of $\mathrm{e}^{+} \mathrm{e}^{-}$ is limited to $\sqrt{s} / 2$. A special case is offered by the scalar partner of the top. Because of the high mass of the latter, mixing in the stop sector can be substantial and it may be that the stop has a very light mass eigenstate. Furthermore, for a particular value (0.99) of the mixing angle between the 'right' and 'left' components, $\widetilde{t}_{R}$ and $\widetilde{t}_{L}$, the lightest mass eigenstate, $\tilde{t}_{1}$, decouples from the $Z^{0}$, and is only produced through the small photon coupling (Drees 90). Under such circumstances, the exclusion power of searches, for $\tilde{t} \rightarrow$ $c \chi^{0}$ essentially, is limited, as shown in Fig. 49 (Keranen 92). Strictly speaking, a stop of $40 \mathrm{GeV}$ is not excluded yet, either by LEP I or by TRISTAN. However, the situation should be clarified already at LEP200, where no such decoupling occurs.

The stop is no doubt a candidate to be carefully watched out for at existing and future machines. Both open stop and scalar stoponium bound states should be considered (Drees 93).

\subsubsection{Sleptons}

This is, on the contrary, a favourite domain of $\mathrm{e}^{+} \mathrm{e}^{-}$machines. Each charged lepton $\ell$ has two charged scalar partners, corresponding to the two weak isospin possibilities, doublet or singlet, $\tilde{\ell}_{L}$ and $\tilde{\ell}_{R}$ : the latter receives less radiative corrections and is expected to be lighter. The channels under study are: $\mathrm{e}^{+} \mathrm{e}^{-} \rightarrow \tilde{\ell} \tilde{\bar{\ell}}, \tilde{\ell} \rightarrow \ell \chi^{0}$ leading to two acoplanar leptons. Such events were not observed at LEP I at the level of $4 \times 10^{-4}$ (Giacomelli 93). 
Assuming, for simplicity, a common mass of $m_{\tilde{\ell}}$ for the two states, the following cross-section is expected at the $Z^{0}$ :

$$
\sigma \sim \frac{1}{8} \beta^{3} \sigma_{\nu \bar{\nu}}
$$

where $\beta^{2}=1-4 m_{\widetilde{\ell}}^{2} / m_{Z}^{2}$, and $\sigma_{\nu \bar{\nu}}$ corresponds to the neutrino final state. LEP I has excluded the production of sleptons up to the kinematical limit. From the $Z^{0}$ invisible width the limit is $m_{\tilde{\ell}}>30.6 \mathrm{GeV}$ if $m_{\tilde{\ell}_{R}} \simeq m_{\tilde{\ell}_{L}}$. LEP200 (Coignet 92) and LCs (Becker 93) will find these particles if they are kinematically accessible.

A particular case is the sneutrino whose production may lead to unobservable final states. Such states can be searched for by the radiative method, as for neutrino counting, but at LEP the statistics of this method are not adequate for a meaningful search. The limits are therefore obtained, at LEP I, from the total and invisible width measurements and can be seen in Table 1. This is another case where the kinematical limit is not reached: the achieved limit is however sufficient to rule out sneutrinos as dark matter candidates.

At $\mathrm{e}^{+} \mathrm{e}^{-}$machines, above the $Z^{0}$, the prospects of identifing the presence of invisible states, such as sneutrinos, are rather low, because of the small rate expected and of the unavoidable background from the three neutrino families. A fortiori no such measurements can be envisaged at hadron machines. This is an embarrassingly weak point for the future of searches.

\section{More on Supersymmetry: a Few Scenarios with R-parity breaking}

R-parity is a new multiplicative quantum number ( +1 for ordinary particles, -1 for their SUSY partners). Its conservation implies that sparticles are produced in pairs, and that a decaying sparticle cascades down to the LSP, which is stable. Up to now, I have assumed such a conservation of R-parity.

However, nature may have chosen a different way. The possibilities span a wide range of cases where sparticles can be produced singly, and the LSP does not have to be stable. Constraints stem from both astrophysics and particle physics, and set limits on the possible extensions, but leave room for quite non-standard ones.

We will simply review a couple of interesting cases, from the purely experimental point of view.

i) An invisible Higgs

R-parity breaking can lead to various scenarios in which the Higgs boson becomes invisible. $\mathrm{e}^{+} \mathrm{e}^{-}$colliders, exploiting kinematical constraints, should be able to deal with such cases. This has been shown by LEP I. Considering the bremsstrahlung process $\mathrm{e}^{+} \mathrm{e}^{-} \rightarrow \mathrm{HZ}$, the final state is $\mathrm{f} \bar{f}+$ missing energy. The exploitation of the $\mathrm{Z}$ $\rightarrow \mathrm{q} \bar{q}$ decay enhances the statistical significance. The ALEPH result (Buskulic 93a) is shown in Fig. 50. A global analysis of LEP data (Lopez-Fernandez 93) (Fig. 51) leads to similar results, and also gives prospects for LEP200. The production of an invisible Higgs boson well below the SM rate can be excluded. Linear colliders should keep this possibility if beamstrahlung and bremsstrahlung are well understood. HCs should participate in this kind of physics as well (Frederiksen 92).

ii) An unstable gluino

An example of a non-conventional model is that of Babu 90: it was invented to give a large transition magnetic moment to neutrinos, so that some features of the solar neutrino signal (probably not relevant anymore) could be explained. 
In this model the LSP is the photino $\tilde{\gamma}$, which can be pair produced by $\tilde{e}$ exchange, and decays to modes violating $\mathrm{R}$-parity, such as $\widetilde{\gamma} \rightarrow \tau \ell \nu$.

OPAL has looked for such events having two leptons in each hemisphere and found none (Acton 93a). This model has nearly been ruled out.

\section{Exotica from Compositeness and extended models}

Compositeness is the assumption that, at some energy scale $\Lambda$, some underlying structure appears, with an interaction characterized by a coupling g. Note that, if $E \sim \Lambda$, it would be possible to scatter these new constituents, as happens with quarks and gluons in hard processes. If $E<<\Lambda$, as it is probably the case now, the new phenomena can be described by some effective Lagrangian which can lead to various anomalies:

i) anomalous decay modes, ii) anomalous electric and magnetic multipoles, iii) excited objects, leptoquarks, and iv) contact terms.

\subsection{Composite Intermediate Vector Bosons}

Some versions of compositeness assume that the IVB are composite objects so that the following could be observed:

i) Anomalous decays

The prediction of some relevant anomalous $Z^{0}$ decays (such as $Z \rightarrow 3 \gamma, \ldots$ ) has been ruled out down to a significant level after a few million $Z^{0}$ s were recorded at LEP (Table 4) (Rubbia 93).

\section{Table 4}

LEP limits for $\mathrm{Z} \rightarrow \gamma \gamma \gamma$ decays at the 95\% CL [from Rubbia 93].

\begin{tabular}{|l|l|}
\hline \multicolumn{1}{|c|}{ Exp. } & $\mathrm{BR}(\mathrm{Z} \rightarrow \gamma \gamma \gamma)$ \\
\hline ALEPH & $<1.9 \times 10^{-5}$ \\
DELPHI & $<2.3 \times 10^{-5}$ \\
L3 & $<8.7 \times 10^{-6}$ \\
OPAL & $<6.6 \times 10^{-5}$ \\
\hline
\end{tabular}

ii) Anomalous couplings

Anomalous boson couplings will be tested in $\mathrm{e}^{+} \mathrm{e}^{-} \rightarrow \mathrm{W}^{+} \mathrm{W}^{-}$reactions at LEP200 (Renard 92, Eichler 92, Bilenky 93), and with greater accuracies at LCs (Barklow 91, Gounaris 94) and HCs (Plothow-Besch 90, Gounaris 93).

Let us call $\Delta g$ a generic anomalous coupling, indicating a departure from the SM one, and possibly related to an anomalous property of the $\mathrm{W}$ (anomalous magnetic dipole, electric quadrupole). Trying to summarize a complicated situation it can be said that:

a) Present accurate measurements already exclude some (de Rujula 91), but not all (Hagiwara 92, Szalapski 92, Hernandez 92), possible deviations observable at LEP200, which are typically at the level of $\Delta g \sim 0.1$ for a single free parameter, the others being fixed at their SM value.

b) The sensitivity ultimately achievable at LCs (typically $\Delta g \sim 0.01$ at the NLC, and better for higher $\mathrm{L}$ and $\sqrt{s}$ ) and at HCs is of the order of the anomalies that can be expected (Einhorn 92) (but 'reasonable' estimates differ by an order of magnitude). It is also of the order of the 'normal' electroweak corrections, estimated to be at the few per mil level (Beenakker 93).

iii) Additional heavy gauge bosons 
They are predicted by many unifying theories. Direct searches for $Z^{\prime}$ production have been performed at HCs excluding such objects up to $300-350 \mathrm{GeV}$ (Del Aguila 93). At LEP, up to now, $Z^{\prime}$ has been looked for through its potential influence on the $\mathrm{Z}$ properties. If there is mixing between these bosons, the $\mathrm{Z}$ line shape will be modified. Stringent limits on the mixing angle have been obtained in different models (Altarelli 91, Langacker 92, Altarelli 93).

It is clear that one of the outstanding promises of HCs, with their large c.m. energy, is to extend these searches of new or excited bosons up to several TeV (Virdee 93, Pauss 94).

\subsection{Composite Fermions}

The existence of three families of fermions is a strong motivation for a substructure. In the case where fermions are composite objects, in principle, the following can be expected:

i) Excited fermions (Bardadin 92, Dittmar 92)

They are assumed to de-excite radiatively, $f^{*} \rightarrow \mathrm{f} \gamma$; excited neutrinos can decay weakly $\nu^{*} \rightarrow \mathrm{eW}, \nu^{*} \rightarrow \nu \mathrm{Z}$. At LEP excited fermions have been excluded up to the masses shown in Table 1 . Whilst their pair production in $\mathrm{e}^{+} \mathrm{e}^{-}$collisions leads to unambiguous mass limits (generally $m \sim \sqrt{s} / 2$ ), single production at $\mathrm{e}^{+} \mathrm{e}^{-}$or hadron machines, and exchange effects, can only give limits on $f / \Lambda$, where $f$ is the coupling, and $\Lambda$ the compositeness scale. The rejection limits have recently been extended by HERA (Wolf 94). Excited quarks are excluded by CDF, in the simplest model, for $90<m_{q *}<570 \mathrm{GeV}(95 \% \mathrm{CL})$ (Coignet 93$)$.

ii) Leptoquarks

Leptoquarks would be a manifestation of a symmetry between quarks and leptons. All high-energy machines so far have put limits on leptoquarks under various assumptions on their quantum numbers and couplings. The most promising types of collision for this purpose are certainly e-p (Wolf 94). We refer to Wolf 94 and Giacomelli 93 for a complete review.

iii) Contact terms (Eichten 83)

All elementary electromagnetic or electroweak processes tested up to now obey the SM rules, with no room for contact terms, i.e. point-like interactions, up to scales of 2-6 TeV for four leptons (Coignet 93). Table 5 shows the less constraining limits for ee $\gamma \gamma$.

\section{Table 5}

Limits on the energy scale $\Lambda$ in $G e V$, at $95 \%$ CL, of ee $\gamma \gamma$ contact interactions from TRISTAN, ALEPH, and both combined [Coignet 93].

\begin{tabular}{|l|rr|cc|}
\hline & \multicolumn{2}{|c|}{$\mathrm{L}, \mathrm{R}$} & \multicolumn{2}{|c|}{$\mathrm{L}+\mathrm{R}$} \\
\hline & \multicolumn{1}{|c|}{$\Lambda^{+}$} & $\Lambda^{-}$ & $\Lambda^{+}$ & $\Lambda^{-}$ \\
\hline TRISTAN & 96 & 84 & 115 & 101 \\
ALEPH & 102 & 109 & 121 & 130 \\
TRISTAN + ALEPH & 114 & 111 & 135 & 132 \\
\hline
\end{tabular}

However, at LEP, data taken on the $Z^{0}$ (purely imaginary) do not provide a good opportunity to feel the interference with a real contact term: sensitivity should improve much at LEP200 and beyond. 
Although there is probably no new underlying physics, the history of this channel illustrates the powerful cross-checks between experiments.

In 1992, the L3 experiment at LEP reported evidence for $\ell^{+} \ell^{-} \gamma \gamma$ events, with a striking clustering of the $2 \gamma$ mass in the $60 \mathrm{GeV}$ region. The initial distribution is shown in Fig. 52 (Adriani 92).

Since then, prompted by this observation, all four experiments have studied these channels, as well as possibly related ones $(q \bar{q} \gamma \gamma, \nu \nu \gamma \gamma, \ldots)$. The set of events observed within the same cuts as for the initial sample are given in Table 6 and Fig. 53 (Wilson 93). The $2 \gamma$ mass value and resolution depend on the procedure used: direct or recoil mass measurement, utilization or not of a kinematical fit, possibly allowing for extra radiation. We must therefore consider that the distribution shown contains a few ambiguous cases.

Table 6

The $\gamma \gamma$ invariant masses and associated errors of the events observed by the four LEP experiments in different channels $(M \gamma \gamma>50 \mathrm{GeV})$.

\begin{tabular}{|c|c|c|c|c|c|c|}
\hline Exp. & Number of Z & $\mathrm{ee} \gamma \gamma$ & $\mu \mu \gamma \gamma$ & $\tau \tau \gamma \gamma$ & $\nu \nu \gamma \gamma$ & $\mathrm{qq} \gamma \gamma$ \\
\hline ALEPH & $1.8 \times 10^{6}$ & $\begin{array}{l}54.7 \pm 1.0 \\
55.6 \pm 0.4 \\
62.8 \pm 0.4 \\
71.3 \pm 0.3\end{array}$ & $\begin{array}{l}59.4 \pm 0.2 \\
63.4 \pm 0.3\end{array}$ & - & $58.5 \pm 1.9$ & $\begin{array}{c}\text { None above } \\
50 \mathrm{GeV}\end{array}$ \\
\hline DELPHI & $1.6 \times 10^{6}$ & $58.7 \pm 0.8$ & $58.5 \pm 0.5$ & - & & $56.3 \pm 1.6$ \\
\hline L3 & $1.6 \times 10^{6}$ & $60.0 \pm 0.6$ & $\begin{array}{l}58.8 \pm 0.6 \\
59.0 \pm 0.6 \\
62.0 \pm 0.6\end{array}$ & - & & $\begin{array}{c}\text { None above } \\
40 \mathrm{GeV}\end{array}$ \\
\hline OPAL & $1.8 \times 10^{6}$ & $\begin{array}{l}50.1 \pm 2.0 \\
53.8 \pm 2.1\end{array}$ & $54.6 \pm 2.1$ & - & & $\begin{array}{l}43.2 \pm 1.7 \\
59.5 \pm 2.2 \\
76.0 \pm 2.6\end{array}$ \\
\hline Total & $6.8 \times 10^{6}$ & & & & & \\
\hline
\end{tabular}

The channels considered have a background caused by double-hard radiation. Several generators seem to agree reasonably well. Figure 54 (Martinez 92) shows the expected rates. In the mass range of Fig. 53, 9 events are expected whilst 15 are observed.

The most conspicuous fact in Fig. 54 is the foreseen absence of the $\nu \nu \gamma \gamma$ radiative channel. The single event observed by ALEPH (Buskulic 93b) compatible with this mode within cuts is therefore of prime importance. If it were a genuine one it would certainly signal the presence of new physics. Unfortunately, it is also compatible with a multi- $\gamma$ event, from which two $\gamma$ were lost at small angles: this illustrates again the importance of strict hermeticity for searches.

Barring this event, the distribution of the observed events among fermions is therefore compatible with the one expected from radiation. On the other hand, it disagrees with the simplest assumption of new physics that we can consider: the associated production $\mathrm{e}^{+} \mathrm{e}^{-} \rightarrow \mathrm{Z}^{*} \mathrm{X}$. For this channel LEP provides the following limit: $B\left(Z \rightarrow \mathrm{Z}^{*} \mathrm{X}\right) B(X \rightarrow$ $\gamma \gamma) \leq 3 \times 10^{-6}$

More can be said about the limits obtained for the $\mathrm{X}$ particle in potentially related channels. The $\mathrm{X}$ production by $2 \gamma$ interactions and its subsequent decay to $2 \gamma$ was searched for and the result is

$$
\Gamma_{X} B^{2}(X \rightarrow \gamma \gamma) \leq 3 M e V
$$


The associated production $\mathrm{e}^{+} \mathrm{e}^{-} \rightarrow \gamma \mathrm{X}, \mathrm{X} \rightarrow \gamma \gamma$ was searched for but no significant accumulation in the $M_{\gamma \gamma}$ plot was observed.

The inclusive study of the reaction $\mathrm{e}^{+} \mathrm{e}^{-} \rightarrow \ell^{+} \ell^{-} \mathrm{X}$, where $\mathrm{X}$ goes to any state different from $2 \gamma$, allowed a limit to be set: $B(\mathrm{Z} \rightarrow \ell \ell \mathrm{X})[1-B(\mathrm{X} \rightarrow \gamma \gamma)] \leq 7 \times 10^{-6}$.

Putting together the limits above, an upper limit on $\Gamma_{X}$ can be obtained. It can therefore be stated that the new object, to be visible with the present integrated luminosity, must be a very narrow one. According to OPAL $\Gamma_{X} \leq 130 \mathrm{MeV}$ (Acton 93b).

All searches performed at TRISTAN to look for such an object were also negative (Matsumoto 93).

The general impression is that probably a normal double-radiation phenomenon is being observed: attention was drawn to the channel by an initial statistical fluctuation. However, it is also fair to say that, at the present level of statistics, and barring preconceived ideas about the features of new physics, a conclusion cannot be draw in either direction.

More statistics are eagerly awaited: it may even be doubted whether the normal LEP I phase will bring enough luminosity to give a definitive answer. We may have here an argument for a high-luminosity run. In any case, the occurrence of a single gold-plated $\nu \nu \gamma \gamma$ candidate would be a decisive fact.

Prior to the LEP results, the existence of an eventual object $X$ in this mass range, to be searched for in the $2 \gamma$ mode, was postulated by Marciano 80, Hatsuda 91, in the frame of the colour sextet model. However, the properties expected a priori for this nonstandard Higgs do not fit well either with what is observed.

\section{Conclusion}

Searches for new particles and new effects at accelerators, present and future, constitute an ever active field. Both aspects - the confrontation of accurate measurements and the direct searches - should be pursued conjointly.

Tristan, the SLC and especially LEP have accomplished a superb programme in both directions: in several sectors of searches, LEP I is nearly reaching its limits whilst in others more luminosity can still help.

The next major steps will be the luminosity rise at HERA and the Tevatron, and the energy rise of LEP. An optimal use of these machines should be made. We recall, in particular, that the energy domain near the end point of LEP200 is a critical one in the quest for SUSY.

As for future colliders, it is clear that, depending on the physics scenario that prevails, HCs and LCs may have quite complementary impacts. The exploration of the vast c.m. energy domain offered by HCs is full of promises, and must absolutely be performed. A positive decision about the realization of the LHC is the next step eagerly awaited for by the high-energy physics community. Linear colliders will pursue the quest for accuracy (Z pole measurements, $m_{t}$, etc.); in a SUSY scenario they may also be the right machine to decipher its properties.

\section{Acknowledgements}

I would like to thank F. Pauss and F. Richard for discussions, and the CERN Desktop Publishing Service, M. Glomet, J. Navarria, and G. Prost, for its efficient work. 
Aachen 87

Abe 91

Abe 93a

Abe 93b

Abe 94

Acton 93a

Acton 93b

Adriani 92

Albajar 87

Alcaraz 93

Alcock 93

Alexander $\mathbf{8 8}$

Altarelli 90

Altarelli 91

Altarelli 93

Alitti 92

Amaldi 91

Amaldi 92

Anselmo 91

Atlas 92

Aubourg 93

Babu 90

Baer 90

Bagger 93

Bagliesi 92

Bai 92

Barbaro Galtieri 93

Barbieri 89

Barbieri 91a

Barbieri 91b

Barbieri 93

Bardadin 92

Bardeen 90

Barger 92

Barklow 91

Barklow 93

Becker 93

Beenakker 93

Berggren 93

Bernreuther 93

Bertolini 91
: ECFA Workshop on LEP200, CERN 87-08, ECFA 87/108 (June 87).

: F. Abe et al., Phys. Rev. D43 (1991) 2070.

: K. Abe et al. (SLD), Phys. Rev. Lett. 70 (1993) 2517.

: F. Abe et al., Phys. Rev. Lett. 70 No. 15 (1993) 2232.

: SLAC Pub 6456 (March 94).

: OPAL Collaboration, Phys. Lett. B313 (1993) 333.

: OPAL Collaboration, Phys. Lett. B311 (1993) 391.

: O. Adriani et al., CERN-PPE/92-152.

: UA1 Collaboration, Phys. Lett. B198 (1987) 261.

: J. Alcaraz et al., preprint CERN-PPE/93-28.

: C. Alcock et al., Nature 365, 621 (1993).

: Polarization at LEP, CERN 86-06 (1986).

: G. Altarelli and R. Barbieri, Phys. Lett. B253 (1990) 161.

: G. Altarelli et al., Phys. Lett. B261 (1991) 146.

: G. Altarelli et al., CERN-TH 6947/93

: J. Alitti et al. (UA2), Phys. Lett. B276 (1992) 354.

: U. Amaldi et al., PL B260, 447 (1991) and references therein.

: U. Amaldi, DELPHI 92-127, Gen 133 (September 92).

: F. Anselmo et al., Nuovo Cimento 104A (1991) 1817.

: Atlas Letter of Intent CERN/LHCC/92-4 (October 92).

: E. Aubourg et al., Nature, 365, 623 (1993).

: K.S. Babu et al., Phys. Rev. D42 (1990) 3778.

: H. Baer et al., Phys. Rev. D41 (1990) 906.

: J. Bagger et al., Fermilab Pub-93/040-T.

: G. Bagliesi et al., preprint CERN-PPE/92-05.

: BES Collaboration, Phys. Rev. Lett. 69 (1992) 3021.

: International Europhysics Conference on High Energy Physics,

Marseille, July 93, talk by L. Barbaro Galtieri.

: R. Barbieri, in Physics at LEP I, CERN 89-08 (1989).

: R. Barbieri et al., IFUP-TH 48/91.

: R. Barbieri et al., Phys. Lett. B258 (1991) 395.

: R. Barbieri et al., preprint CERN-TH.6830/93.

: M. Bardadin-Otwinowska, Z. Phys. C55 (1992) 163.

: W. Bardeen et al., Phys. Rev. D41 (1990) 1647.

: V. Barger et al., Phys. Rev. D45 (1992) 1484.

: T. L. Barklow, in 'Physics and experiments with linear Colliders, Saariselka, Finland (September 91), eds, R. Orava et al.

(World Scientific Singapore, 92).

: T.L. Barklow, SLAC Pub-6280 (1993).

: R. Becker, C. Vander Velde, IISN 0379-301X (October 93).

: W. Beenakker and A. Denner, preprint CERN-TH.6928/93, DESY-93-079.

: M. Berggren et al., DELPHI 93-59 Phys. 287 (1993).

: W. Bernreuther and T. Schroder, DESY 93-163, November 93.

: S. Bertolini et al., Nucl. Phys. 353 (1991) 591, and 294 (1987) 321. 
Bertolini 94

Besson 94

Bilenky 93

Blondel 91

Blucher 91

Boos 93

Borzumati 93

Bouchiat 84

Brodsky 93

Buskulic 93a

Buskulic 93b

Camilleri 92

Campos 93

Casalbuoni 85

Casalbuoni 94

Chanowitz 92

Chen 93

CMS 92

Coignet 92

Coignet 93

Dawson 89

de Boer 94

de Rujula 91

del Aguila 93

Dittmar 92

Dobado 90

Drees 90

Drees 93

Drees 94

Dubinin 93

Dusedau 86

Eichler 92

Eichten 83

Einhorn 92

Elliott 92

Ellis 90

Ellis 91

Ellis 93a

Ellis 93b

Ellwanger 93

Espinosa 92

Fahri 81

Fayet 77

Fayet 93

Ferrara 74

Frary 93
: S. Bertolini and F. Vissani, SISSA 40/94/EP (March 94).

: D.Z. Besson, CLNS 93-1259.

: M. Bilenky et al., Nucl. Phys B409 (1993) 22.

: A. Blondel et al., LAPP-TH.343/91.

: High Luminosity at LEP, CERN 91-02 (1991), E. Blucher et al., (eds).

: E. Boos et al., DESY 93-183 (December 93).

: F.M. Borzumati, DESY 93-090 (August 93).

: M.A. Bouchiat, L. Pottier, Science 234, p. 1203.

: S. Brodsky, SLAC-Pub-6314 (1993).

: D. Buskulic et al., Phys. Lett B313 (1993) 312.

: D. Buskulic et al., CERN-PPE/93-75, April 1993.

: Workshop on LEP200, LEPC (November 92), talk by L. Camilleri.

: F. de Campos and J.W.F. Valle, FTUV/93-9.

: R. Casalbuoni et al., Phys. Lett. B155 (1985) 55.

: R. Casalbuoni et al., UGVA-DTP 1994/03-845 (March 94).

: M.S. Chanowitz, LBL-32846 (1992), LBL-31651 (1992), LBL-32938 (1992).

: P. Chen et al., SLAC-PUB-5873 (April 1993).

: CMS Letter of Intent CERN/LHCC 92-3 (October 92).

: Workshop on LEP200, LEPC (November 92), talk by G. Coignet.

: G. Coignet, Invited talk at Cornell Int. Conf., LEPPH093, August 1993.

: The Higgs Hunter Guide, UCD-89-4, SCIPP-89/13, BNL-41644.

: W. de Boer et al., IEKP-KA/94-05.

: A. de Rujula et al., preprint CERN-TH.6272/91. Nucl. Phys.

B384 (1992) 3.

: F. del Aguila et al. UPR-0583T.

: M. Dittmar, CERN-PPE/92-177 (Oct. 92).

: A. Dobado et al., CERN/TH-5670/90.

: M. Drees and K. Hikasa, KEK-TH-262, preprint CERN-TH.5854/90.

: M. Drees and M.M. Nojiri, MAD/PH/808. KEK-TH-379.

: M. Drees, MAD/PH/818, February 1994.

: M. Dubinin et al., KEK Preprint 93-138 (October 93).

: D.W. Dusedau and J. Wudka, Phys. Lett. B, Vol. 180 No 3 (1986) 290.

: Workshop on LEP200, LEPC (November 92), talk by R. Eichler.

: E. Eichten et al., Phys. Rev. Lett. 50 (1983) 811.

: M.B. Einhorn and J. Wudka, UM-TH/92-25.

: T. Elliott et al., SHEP-92/93-21.

: J. Ellis et al., Phys. Lett. B249 (1990) 441.

: J. Ellis et al., Phys. Lett. B257 (1991) 83 and B262 (1991) 477.

: J. Ellis, preprint CERN-TH.7125/93.

: J. Ellis et al., preprint CERN-TH.6824/93.

: U. Ellwanger HD-THEP-93-4 (February 93).

: J.R. Espinosa and M. Quiros, IEM-FT-60/92 (August 92).

: E. Farhi and L. Susskind, Phys. Rep. 74 (1981) 277 and references therein.

: P. Fayet and S. Ferrara, Phys. Rep. 32C (1977) 249.

: P. Fayet, talk at the Rencontres du Vietnam, Hanoi, Vietnam, December 93.

: S. Ferrara and E. Remiddi, Phys. Lett. B53 (1974) 347.

: M.N. Frary and D.J. Miller, DESY 92-123A. 
Frederiksen 92 : S. Frederiksen et al., SSCL-577 (1992).

Froidevaux 93 : D. Froidevaux, CERN/PPE/93-148 (June 93).

Fujii 93

: K. Fujii et al., KEK Preprint 93-125 (October 93).

Gaillard 92 : M.K. Gaillard LBL-32389 (1992).

Georgi 74 : H. Georgi, S. Glashow, Phys. Rev. Lett. 32 (1974) 438.

Giacomelli 93 : G. Giacomelli and P. Giacomelli, Riv. Nuovo Cimento 16, serie 3, No. 3 (1993), preprint CERN-PPE/93-107.

Gilbert 86 : S.L. Gilbert and C.E. Wieman, Phys. Rev. A34 (1986) 792.

Ginzburg 82 : I. Ginzburg et al., JETP 34 (1982) 491.

Glover 89 : E. Glover et al., preprint CERN-TH.5584/89.

Gounaris 93 : G.J. Gounaris and F.M. Renard PM/93-02.

Gounaris 94 : G.J. Gounaris and F.M. Renard PM/94-03.

Grivaz $91 \quad$ : J.F. Grivaz, LAL 91-63.

Grivaz 92 : J.F. Grivaz, LAL 92-59.

Grivaz 93 : J.F. Grivaz, LAL 93-11.

Gross 92 : E. Gross and P. Yepes, preprint CERN-PPE/92-153.

Guignard 93 : G. Guignard, in Physics and experiments with linear colliders, Waikoloa, Hawaii. eds. F. A Harris et al., World Scientific.

Haber 85 : H. Haber and G. Kane, Phys. Rep. 75 (1985) 117.

Haber 91 : H.E. Haber and R. Hempfling Phys. Rev. Lett. 66 (1991) 1815.

Haber 92 : H.E. Haber, SCIPP-92-31.

Hagiwara 91 : K. Hagiwara, in 'Physics and experiments with linear colliders, Saariselka, Finland (September 91), eds. R. Orava et al., (World Scientific Singapore, 92).

Hagiwara 92 : K. Hagiwara et al., Phys. Lett. B283 (1992) 353.

Hagiwara 93 : K. Hagiwara et al., KEK-TH-370 (August 93).

Harari $84 \quad$ : H. Harari, Phys. Rep. 104, (1984) 159.

Hatsuda 91 : T. Hatsuda and M. Umezawa, Phys. Lett. B254 (1991) 493.

Hebbeker 93 : T. Hebbeker, preprint CERN-PPE/93-89.

Hempfling 93 : R. Hempfling and A.H. Hoang, DESY 93-162 (November 93).

Hernandez 92 : P. Hernandez et al., preprint CERN-TH.6670.

Heusch $93 \quad$ : C. Heusch, in Physics and experiments with linear colliders, Waikoloa, Hawaii. eds. F. A Harris et al., World Scientific.

Hewett 93 : J.L. Hewett, Phys. Lett. Rev. 70 (1993) 1045.

Hikasa $91 \quad$ : K. Hikasa, in 'Physics and experiments with linear colliders, Saariselka, Finland (September 91), eds. R. Orava et al., (World Scientific Singapore, 92).

Hildreth 93 : M.D. Hildreth, SLAC Pub. 6036 (1993).

Iarocci 94 : E. Iarocci, Academic Training at CERN on new detector developments, March 1994.

Iwata 94 : S. Iwata, KEK P 93-157.

Janot $92 \quad:$ P. Janot, LAL/92-27.

Janot 93 : P. Janot, LAL 93-28 (July 93).

Jezabek 93 : M. Jezabek and J.H. Kuhn, Phys. Lett. B301 (1993) 121.

Jikia 93 : G.V. Jikia, Nucl. Phys. B405 (1993) 24.

JLC 92 : KEK Report 92-16 (December 92).

Kane 93 : G.L. Kane et al., UM-TH-93-24.

Keranen 92 : R. Keranen et al., DELPHI 92-172, Phys. 255. 
Kinoshita 93

Kodaira 93

Kuhn 93

Kunszt 91

Langacker 81

Langacker 87

Langacker 92

Langacker 91a

Langacker $91 \mathrm{~b}$

Langacker 94

Layssac 93

Lefrançois 93

LEP 94

Lopez-Fernandez 93

L3P 92

Luty 93

Lyons 83

Marciano 80

Martinez 92

Matsumoto 93

Nilles 84

Okada 91

Olechowski 92

Omori 93

Pauss 90

Pauss 94

Peskin 85

Peskin 90

Peskin 91

Plothow-Besch 90

Prescott 80

Pretzl 93

Renard 92

Richard 91

Romao 92

Ross 84

Rubbia 93
: T. Kinoshita, CLNS 93-1186.

: J. Kodaira et al., HUPD-9316 (November 93).

: J. Kuhn, in Physics and experiments with linear colliders,

Waikoloa, Hawaii. eds. F. A Harris et al., (World Scientific,)

: Z. Kunszt and F. Zwirner, preprint CERN-TH.6150/91, ETH-TH.91-7.

: P. Langacker Phys. Rep. 72C (1981) 185.

: P. Langacker et al., Rev. Mod. Phys. 64, 87.

: P. Langacker et al., Phys. Rev. D45 (1992) 278.

: P. Langacker and M. Luo, Phys. Rev. D44 (1991) 817.

: P. Langacker et al., UPR 458T (February 91).

: P. Langacker and N. Polonsky, UPR-0594T, February 1994.

: J. Layssac et al., PM/9304.

: J. Lefrançois, LAL 93-6.

: Combined electroweak results of LEP experiments, LEPEWWG/94-01.

: A. Lopez-Fernandez et al., Phys. Lett. B312 (1993) 240.

: L3P Letter of Intent CERN/LHCC 92-5 (September 92).

: M.A. Luty et al., Phys. Lett. Rev. 70 No. 3 (1993) 529.

: L. Lyons, Prog. Part. \& Nucl. Phys. 10 (1983) 227.

: W.J. Marciano, Phys. Rev. D21 (1980) 2425.

: M. Martinez and R. Miquel, preprint CERN-PPE/92-211.

: S. Matsumoto KEK-TH-357.

: H.P. Nilles, Phys. Rep. 1 (1984) 110.

: Y Okada et al., Prog. Theor. Phys. 85 (1991) 1.

: M. Olechowski and S. Pokorski, MPI-Ph/92-118.

: T. Omori, KEK P 93-121 (1993).

: F. Pauss, in LHC Workshop, Aachen, CERN/90-10 (October 90).

: F. Pauss, talk at the Rencontres du Vietnam, Hanoi, Vietnam,

December 93. Preprint ETHZ-ITP PR-94-8.

: M.E. Peskin, in Proc. Kyoto Conf., August 1985.

: M.E. Peskin and T. Takeuchi, Phys. Rev. Lett. 65 (1990) 964.

: M.E. Peskin, in Physics and experiments with linear colliders, Saariselka, Finland (September 91), eds. R. Orava et al. (World Scientific Singapore 92).

: H. Plothow-Besch, in LHC Workshop, Aachen, CERN/90-10, 2, 91, (October 90).

: C.Y. Prescott, Proc. 1980 Int. Symp. on High-Energy Physics with

Polarized Beams and Polarized Targets, Lausanne, 1980, eds.

C. Joseph and J. Soffer (Birkhäusser Verlag, Basel, 1981), p. 34.

: K.P. Pretzl, Preprint Bern BUHE-93-3.

: F.M. Renard, PM/92-28 and references therein.

: F. Richard, LAL 91-62 (November 91).

: J.C. Romao et al., FTUV/92-39.

: G.G. Ross, Grand Unified Theories, Benjamin Inc. (1984).

: A. Rubbia, Search for New Physics at LEP, talk given at the Paris Conf. on Neutral Currents: Twenty Years After (1993). 
Sadoulet 92 : B. Sadoulet, in Particle Astrophysics, Fourth Rencontres de Blois (Ed. Frontières, Blois, 1992).

Schmelling 93 : M. Schmelling et al., CERN/PPE/93-193.

Siemann 94 : R.H. Siemann, SLAC-PUB 6417 (1994).

Sjostrand 93 : T. Sjostrand and V. Khose, preprint CERN-TH.7011/93.

Sopzcak 93 : A. Sopzcak, in Physics and experiments with linear colliders, Waikoloa, Hawaii. eds. F. A Harris et al. (World Scientific,) CERN PPE/93-197.

Strassler 90 : M.J. Strassler and M.E. Peskin, SLAC Pub 5308 (September 90).

Susskind 79 : L. Susskind, Phys. Rev. D20 (1979) 2619.

Szalapski 92 : R. Szalapski et al., MAD/PH/719.

Tabarelli 93 : International Europhysics Conference on High Energy Physics, Marseille, July 93 , talk by T. Tabarelli.

Telnov $93 \quad$ : V. Telnov, in Physics and experiments with linear colliders,

Waikoloa, Hawaii, eds. F. A Harris et al. (World Scientific,)

Treille $93 \quad$ : D. Treille, preprint CERN-PPE/93-54 Rev.

Turner 93 : M.S. Turner, Fermilab-Conf. 92/382.A, January 93.

Valle $92 \quad$ : J.W.F. Valle, FTUV/92-44.

Virdee 93 : J. Virdee, CMS TN/94-132.

Watanabe 93 : I. Watanabe, HUPD-9301, Jan. 93.

Weinberg 79 : S. Weinberg, Phys. Rev. D19 (1979) 1277.

Wiik $93 \quad$ : B. Wiik, in Physics and Experiments with Linear Colliders,

Waikoloa, Hawaii. F. A Harris et al., eds. World Scientific.

Wilson 93 : International Europhysics Conference on High Energy Physics, Marseille, July 93 , talk by G. Wilson.

Wolf 94 : G. Wolf, DESY 94-022 (1994).

Zerwas 91 : P. Zerwas, in 'Physics and experiments with linear Colliders, Saariselka, Finland (September 91), R. Orava et al., (eds), World Scientific (Singapore) 92'.

Zisman 94 : M.S. Zisman, LBL 34556 (1994).

Zwirner 93 : F. Zwirner, The SUSY World, preprint CERN-TH.6051/93 and references there of. 


\section{APPENDIX 1}

\section{THE STANDARD MODEL}

The Standard Model incorporates electromagnetic and weak interactions, unified into the electroweak one, and concerns the strong interaction as well. The Standard Model provides a classification of the basic constituents of which all matter is built, and a common description of all interactions.

\section{Constituents}

The basic constituents are fermions ( $\operatorname{spin} \frac{1}{2}$ ), leptons, which are freely propagating particles, and quarks which are 'confined' objects, always bound to other quarks or antiquarks. Single quarks are not detectable directly, but their presence can sometimes be inferred from the appearance of a jet of particles. Leptons and quarks are grouped into families, as shown in the Table A1. Three such families are known. Measurements of the $\mathrm{Z}^{0}$ boson properties show that there are no further families, at least with the pattern of the first three, with a light neutrino. There is no explanation at present for the existence of three families. The first one is sufficient to build the present Universe, including ourselves. However, constituents belonging to all three families are produced in energetic collisions, and these were conditions prevailing in the early moments of the Universe. There is a basic asymmetry, visible in the Table, between left-handed and right-handed fermions. Left-handed leptons appear as doublets, and right-handed ones as singlets (the opposite is true for antileptons). Doublets and singlets can be considered as mathematical objects in an internal space called the weak isospin space.

\section{Forces}

All fundamental forces arise from the exchange of a vector boson (spin 1) between two fermions: the photon for the electromagnetic interaction; the $Z^{0}$ and $W^{ \pm}$for the weak interactions; and the gluons (which, like quarks, never appear singly) for the strong interactions. Each boson couples to a charge carried by the constituents: the photon couples to the electric charge, the $\mathrm{W}$ to the weak charge, and the gluons to a charge called 'colour' specific of strong interactions. Whilst the photon does not itself carry the electric charge, the weak bosons and the gluons do carry the charges for the weak and colour interactions, respectively. The weak bosons can therefore interact between themselves, and this novel property will be studied, in particular at LEP200. The self-interactions of the gluons are the source of the surprising phenomenon of confinement.

\section{Processes}

The simple diagram of Fig. A1.1 is thus the prototype of all processes studied in HE physics, that one obtains by varying the nature of external and internal lines. However higher order processes, involving in particular loops of virtual particles as in Fig. A1.2, intervene as corrections to the first order 'tree level' one and make the situation both more complicated and more interesting. Under special circumstances, these loops allow to feel the presence of particules too heavy to be directly produced, as the top quark by measuring accurately the $Z^{0}$ properties at LEP I. What one 'sees' as loops and in loops depends on the resolution power of the observation: one can then expect that the strength of the interaction will slightly vary with the resolution power and thus with the energy, so that constants measuring this strength $\left(\alpha, \alpha_{s}, \ldots\right)$ are actually 'running' with energy. This 
is indeed the case. But, due to the difference in the populations of particles appearing in the loops, the various interactions behave differently: while the e.m. and weak have a strength increasing with energy, it is the opposite for the strong interaction. This fact goes in the direction of a possible merging at very high energy.

\section{The Intermediate Vector Bosons}

In the SM the electroweak interaction is carried by four bosons, $\mathrm{W}^{ \pm}, \mathrm{W}^{0}$ and $\mathrm{B}^{0}$. The first three are associated to rotations in the weak isospin space, changing a fermion into another one and belonging to the group $\mathrm{SU}(2) . \mathrm{B}^{0}$ is associated to the familiar electromagnetic phase rotation [group U (1)]. The corresponding coupling constants of bosons to fermions are noted $\mathrm{g}$ and $\mathrm{g}^{\prime}$, respectively. In the neutral sector, the physical bosons are not the states $\mathrm{W}^{0}$ and $\mathrm{B}^{0}$, but the photon and the $\mathrm{Z}^{0}$, derived from these by a rotation defined below.

\section{Grand Unification}

Grand Unification extends the Standard Model by asserting that all three interactions are unified into a single one at very high energy (relevant to the very early moments of the Universe). This seems to be the case, at least for a supersymmetric version of the SM, as we discuss elsewhere, and the unification energy turns out to be about $10^{16}$ $\mathrm{GeV}$. Below this energy the single interaction is broken into two separate interactions, the strong and the electroweak. The breaking of the electroweak symmetry, on the other hand, is 'next door' in energy, around the so-called Fermi scale $(\sim 300 \mathrm{GeV})$, below which we see the familiar electromagnetic and weak interactions. Present and future machines, including LEP200, will allow us to explore the domain of electroweak symmetry breaking.

\section{The Higgs mechanism}

The breaking of electroweak symmetry is, in the Standard Model, caused by the Higgs mechanism, which is similar to the superconductivity phenomenon. In a superconductor there is a field of Cooper pairs, which repels external magnetic fields (Meissner effect). Magnetic fields can only penetrate it to a depth $\ell$. It is as if, in the superconductor, the photon has acquired a mass $\sim \ell^{-1}$ related to the density of Cooper pairs. The Higgs field is an analog of the field of Cooper pairs, but in a very different context where the weak isospin space replaces the usual one dimensional space of electromagnetism. The Higgs field, a complex doublet (4 components) in the weak isospace, is supposed to lead to an analogue of the Meissner effect: the vector bosons $Z^{0}$ and $W^{ \pm}$acquire a mass related to the Higgs field density which is called the Higgs vacuum expectation value and denoted $v$. This is a well defined quantity: $v=2^{-3 / 4} G_{F}^{-1 / 2}$ with $G_{F}^{-1 / 2} \simeq 300 \mathrm{GeV}$. Of the four degrees of freedom of the Higgs field, three are used in that operation by which the three vector bosons get a longitudinal component, as any spin 1 massive particle. One degree of freedom of the Higgs field is left, corresponding to the existence of a neutral scalar particle, the Higgs boson. After symmetry breaking, the photon, which has stayed massless, and the $\mathrm{Z}^{0}$, which acquired a mass of $\sim 91 \mathrm{GeV}$, are very different particles indeed, in spite of their original close relationship.

In terms of already defined parameters $m_{W}=1 / \sqrt{2} g \mathrm{v}$. As for the Higgs mass, it can be written $m_{H}=\lambda v$, where $\lambda$ is a constant appearing in the Higgs potential and of unknown value: the mass is therefore unknown as well. To make sense as a particle the Higgs boson must be lighter than $\sim 1 \mathrm{TeV}$. Theoretical considerations lead us to expect a lower limit for its mass, depending on $m_{t}$, and an upper limit, depending on the scale of new physics and necessary to avoid the problem of triviality quoted in the text. If there 
is no new physics up to the GU scale, $m_{H}<200 \mathrm{GeV}$.

The Higgs mechanism provides the mass of all particles, expressed as

$$
m_{x}=\lambda_{x} v
$$

where $\lambda_{x}$ is the so-called Yukawa coupling of the species $x$ to the Higgs boson. A high mass implies a large Yukawa coupling, and therefore a large coupling between the Higgs and the $x$ state. This explains why the Higgs boson is expected to decay into the heaviest objects available and whose production is allowed by energy conservation.

\section{SM parameters}

In the Standard Model all quantities can be computed at first order from the three basic ones $g, g^{\prime}$ and $v$. But, motivated by the accuracy with which they are experimentally known, one usually uses instead an equivalent triplet: the fine-structure constant $\alpha$, the weak coupling constant $G_{\mu}$, and the $Z^{0}$ mass $M_{Z}$. We already explained that at higher orders other parameters are relevant as well, like $m_{\text {top }}, m_{H i g g s}$ and $\alpha_{s}$.

A very important, albeit derived, quantity in the Standard Model is the weak mixing angle $\theta_{W}$. It is the angle of rotation which relates the two basic orthogonal neutral states of the electroweak theory, $\mathrm{W}^{0}$ and $\mathrm{B}^{0}$, to the physical states, the $\gamma$ and the $\mathrm{Z}^{0}$. This angle (or rather $\sin ^{2} \theta_{W}$ ) appears in the expressions of most of the Standard Model observables, and can be extracted from their measurements. Checking the Standard Model amounts to checking that all values of $\sin ^{2} \theta_{W}$, extracted from the various observables, coincide within errors. The error on $\sin ^{2} \theta_{W}$ can be considered as a measure of the quality or sensibility of the observable.

\section{Chromodynamics}

This is the modern version of the strong interaction. Quarks interact between themselves by exchanging gluons. Gluons are coupled to the colour charge of the quarks: they actually exchange color between quarks and therefore carry this charge and behave as tensors in colour space. This property leads to a peculiar behaviour of the strong coupling, $\alpha_{s}$, as a function of the resolution power and energy. At large energy, $\alpha_{s}$ decreases towards zero; at small energy or large distance, $\alpha_{s}$ increases in such a way that the separation of quarks cannot occur (confinement). In the chromodynamics Lagrangian nothing forbids the existence of a $\mathrm{CP}$ violating term, involving the parameter $\theta_{Q C D}$ : however this parameter seems, for an unknown reason, to be zero to a high level of accuracy. 
Table A1

\begin{tabular}{|ccccc|}
\hline \multicolumn{3}{|c|}{ Lepton and Quark Families } \\
\hline & $\begin{array}{c}\text { First } \\
\text { Family }\end{array}$ & $\begin{array}{c}\text { Second } \\
\text { Family }\end{array}$ & $\begin{array}{c}\text { Third } \\
\text { Family }\end{array}$ \\
Leptons & $\left(\begin{array}{c}\nu_{e} \\
e\end{array}\right)_{L H}$ & $\left(\begin{array}{c}\nu_{\mu} \\
\mu\end{array}\right)_{L H}$ & $\left(\begin{array}{c}\nu_{\tau} \\
\tau\end{array}\right)_{L H}$ & doublet \\
Left-Handed (LH) & $(\mathrm{e})_{R H}$ & $(\mu)_{R H}$ & $(\tau)_{R H}$ & singlet \\
Right-Handed (RH) & $\left(\begin{array}{c}u \\
d\end{array}\right)$ & $\left(\begin{array}{c}c \\
s\end{array}\right)$ & $\left(\begin{array}{c}t \\
b\end{array}\right)$ & \\
\hline Quarks & & \\
\end{tabular}

Figure A1.1: 'Tree level' diagram.

Figure A1.2: Higher order 'loop' diagram. 


\section{APPENDIX 2}

\section{SUPERSYMMETRY}

Supersymmetry (SUSY) establishes a link between the known particles and hypothetical superpartners whose spin differs by half a unit. A fermion is associated to a boson (and vice-versa). Since there is no evidence up to now of this mirror population, one assumes that supersymmetry is a broken symmetry.

As we mentioned in the text an encouragement toward SUSY is the convergence of the three coupling constants at very high energy in the supersymmetric scenario.

One of the main virtues of supersymmetry is to explain naturally the stability of the mass of a light scalar like the Higgs boson. Owing to virtual processes such as those in Fig. A2, the mass of these objects would be driven towards the highest conceivable scales (Grand Unification or Planck Scale). The addition of the boson loops of Fig. A2 cancels this divergence and ensures mass stability. However, this cancellation is effective only if the mass of the superpartners is not too high, say below $\sim 1 \mathrm{TeV}$.

Apart from these considerations, the masses of the superpartners are not known a priori. An exception occurs in the SUSY Higgs sector where sharp bounds exist (see later).

Contrary to their masses, the couplings of SUSY particles to ordinary particles and to themselves are perfectly determined: they are the same as for corresponding normal particles, although mixing may eventually complicate the issue.

It has not been possible to relate existing particles among themselves by supersymmetry: this implies that superpartners should all be new undiscovered ones. The minimum expected spectrum of sparticles is given in Table A2. This is the content assumed for the minimal supersymmetric model (MSSM).

\section{R-parity}

In most versions of SUSY one assumes the existence and conservation of a new quantity, the R-parity, a multiplicative quantum number, which is +1 for ordinary particles and -1 for their superpartners.

This conservation law implies:

- that sparticles are produced in pairs;

- that a sparticle decays by cascading down to the lightest one (LSP);

- that the LSP is stable and, being weakly interacting, escapes detection.

This set of properties is usually assumed to be valid for SUSY. However, versions of the theory which incorporate R-parity breaking have been considered as well: in these versions, care must be taken to avoid nucleon decay at a rate exceeding the experimental upper limit.

\section{Symmetry breaking}

In order to avoid the introduction of quadratic divergences, one has to restrict the symmetry breaking terms in the SUSY Lagrangian to a special class: the soft breaking terms. In such conditions, besides the three coupling constants $\alpha_{i}$, there are in the theory five mass parameters:

$$
m_{1 / 2}(\text { or } M), \quad m_{0}, \quad \mu, \quad A, \quad B \text {. }
$$


At the GUT scale all scalar masses are equal to $m_{0}$, all gaugino masses to $m_{1 / 2}$ and all couplings to $\alpha_{G U T}$. The parameter $\mu$ plays an important role in the shape of the Higgs potential and the way breaking occurs.

In the MSSM what happens at the electroweak scale is assumed to be governed, through the running of the parameters expressed by the renormalization group equations (RGE), by the values they take at the GUT scale. The top mass intervenes considerably in the running of the parameters. The SUSY mass spectrum is then much constrained.

\section{Sfermions}

The squarks and sleptons have all the same mass at the GUT scale. In the 'runningdown' squarks get additional radiative corrections from virtual gluons and are therefore heavier than sleptons at low energies. One distinguishes $\mathrm{L}$ and $\mathrm{R}$ squarks and sleptons, where these subscripts do not indicate the helicity (they have no spin) but whether they are $\mathrm{SU}(2)$ doublets or singlets. The L variety gets more radiative corrections and is expected to be heavier than the $\mathrm{R}$ one.

The mass eigenstates are mixtures of the $\mathrm{L}$ and $\mathrm{R}$ weak interaction states: the mixing is proportional to the Yukawa coupling, and therefore may be very relevant in the case of the stop.

\section{Gauginos}

For the gaugino masses, the RGE equations are simple and lead to the following expectations at the weak scale

$$
\begin{aligned}
M_{3}(\tilde{g}) & \approx 2.7 m_{1 / 2} \\
M_{2}\left(M_{Z}\right) & \approx 0.8 m_{1 / 2} \\
M_{1}\left(M_{Z}\right) & \approx 0.4 m_{1 / 2}
\end{aligned}
$$

where the $M_{i}$ are the gaugino masses of the U(1), SU(2) and $\mathrm{SU}(3)$ groups. The gluinos are heavier because they get corrections from the strong coupling constant.

Gauginos mass eigenstates are actually mixtures of the weak eigenstates: they are called charginos and neutralinos for the charged and neutral fields, respectively.

The four neutralino mass eigenstates are $\chi^{0}, \chi^{0 \prime}, \chi^{0 \prime \prime}, \chi^{0 \prime \prime \prime}$ in order of increasing masses. Their nature depends on the values of the SUSY parameters. If for instance $M_{1}, M_{2}, \mu \gg M_{Z}$, the mixing is suppressed and the LSP is a pure state (bino-like).

\section{Higgs sector}

A most constrained domain of SUSY is its Higgs sector: this is developped in the text. The striking fact is the link existing by construction between the mass of Higgs bosons ( 5 of them in the simplest versions, including the MSSM, which deal with two Higgs doublets), and the electroweak boson mass. In particular, at tree level, the lightest scalar, $h^{0}$, must be lighter than the $\mathrm{Z}^{0}$. Actually radiative corrections due to virtual loops of top and stop push this mass slightly upwards, and this depends on the model considered. In the MSSM the two relevant parameters are generally chosen as $m_{A}$, the mass of the pseudoscalar Higgs boson, and $\operatorname{tg} \beta \equiv v_{1} / v_{2}$, the ratio of the vacuum expectation values of the two doublets. 
Table A2 (From Giacomelli 93)

\begin{tabular}{|c|c|c|c|c|c|c|}
\hline \multicolumn{3}{|c|}{ Particles, $R=+1$} & \multicolumn{4}{|c|}{ Sparticles, $R=-1$} \\
\hline Particle & Spin & Charge & Sparticle & Spin & S-name & $\begin{array}{c}\text { Mass } \\
\text { eigenstates }\end{array}$ \\
\hline $\mathrm{e}$ & $1 / 2$ & -1 & $\tilde{e}_{L}, \tilde{e}_{R}$ & 0 & selectron & \\
\hline$\mu$ & $1 / 2$ & -1 & $\tilde{\mu}_{L}, \tilde{\mu}_{R}$ & 0 & smu & \\
\hline$\tau$ & $1 / 2$ & -1 & $\tilde{\tau}_{L}, \tilde{\tau}_{R}$ & 0 & stan & \\
\hline$\nu=\nu_{e}, \nu_{\mu}, \nu_{\tau}$ & $1 / 2$ & 0 & $\tilde{\nu}$ & 0 & sneutrino & \\
\hline $\mathrm{q}=\mathrm{u}, \mathrm{d}, \mathrm{s}, \mathrm{c}, \mathrm{b}, \mathrm{t}$ & $1 / 2$ & $2 / 3,-1 / 3$ & $\tilde{q}_{L}, \tilde{q}_{R}$ & 0 & squark & \\
\hline g & 1 & 0 & $\tilde{g}$ & $1 / 2$ & gluino & \\
\hline$\gamma$ & 1 & 0 & $\tilde{\gamma}$ & $1 / 2$ & photino & \\
\hline$Z^{0}$ & 1 & 0 & $\tilde{Z}$ & $1 / 2$ & zino & neutralinos \\
\hline $\left.\mathrm{H}_{1}^{0}, \mathrm{H}_{2}^{0} h^{0}, H^{0}, A^{0}\right)$ & 0 & 0 & $\tilde{H}_{1}^{0}, \tilde{H}_{2}^{0}$ & $1 / 2$ & neutral Higgsinos & $\left(\tilde{\chi}^{0}-\tilde{\chi}^{0 \prime \prime \prime}\right)$ \\
\hline $\mathrm{H}^{ \pm}$ & 0 & \pm 1 & $\tilde{H}^{ \pm}$ & $1 / 2$ & charged Higgsino & charginos \\
\hline$W^{ \pm}$ & 1 & \pm 1 & $\tilde{W}^{ \pm}$ & $1 / 2$ & wino & $\left(\tilde{\chi}_{1}^{ \pm}, \tilde{\chi}_{2}^{ \pm}\right)$ \\
\hline G & 2 & 0 & $\tilde{G}$ & $3 / 2$ & gravitino & \\
\hline
\end{tabular}

\begin{tabular}{|c|c|c|c|}
\hline & \multicolumn{3}{|c|}{ Spin } \\
\cline { 2 - 4 } & 0 & $1 / 2$ & 1 \\
\hline & $\tilde{\ell}$ & $\ell$ & \\
Particles & $\tilde{q}$ & $\mathrm{q}$ & \\
and & $\mathrm{H}_{1}^{0}, H_{2}^{0}$ & $\tilde{\chi}^{0}-\tilde{\chi}^{0 \prime \prime \prime}$ & $\gamma, Z^{0}$ \\
Sparticles & $\mathrm{H}^{ \pm}$ & $\tilde{\chi}_{1}^{ \pm}, \tilde{\chi}_{2}^{ \pm}$ & $\mathrm{W}^{ \pm}$ \\
\hline
\end{tabular}

Figure A2: Corrections to the Higgs self-energy from Yukawa type interactions. 